NASA/CR-1998-208702

ICASE Report No. 98-39

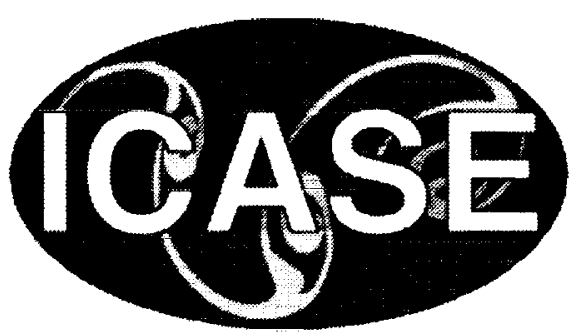

\title{
Numerical Study of Rotating Turbulence with External Forcing
}

P.K. Yeung

Georgia Institute of Technology, Atlanta, Georgia

Ye Zhou

IBM, Yorktown Heights, New York

and

ICASE, Hampton, Virginia

Institute for Computer Applications in Science and Engineering NASA Langley Research Center

Hampton, VA

Operated by Universities Space Research Association

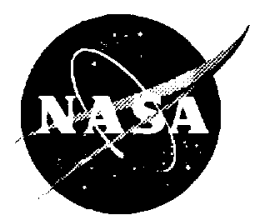

National Aeronautics and Space Administration

Langley Research Center

Hampton, Virginia 23681-2199
Prepared for Langley Research Center under

Contracts NAS1-19480 and NAS1-97046 
Available from the following:

NASA Center for AeroSpace Information (CASI) 7121 Standard Drive

Hanover, MD 21076-1320

(301) 621-0390
National Technical Information Service (NTIS)

5285 Port Royal Road

Springfield, VA 22161-2171

(703) $487-4650$ 


\title{
NUMERICAL STUDY OF ROTATING TURBULENCE WITH EXTERNAL FORCING
}

\author{
P.K. YEUNG* AND YE ZHOU ${ }^{\dagger}$
}

\begin{abstract}
Direct numerical simulations at $256^{3}$ resolution have been carried out to study the response of isotropic turbulence to the concurrent effects of solid-body rotation and numerical forcing at the large scales. Because energy transfer to the smaller scales is weakened by rotation, energy input from forcing gradually builds up at the large scales, causing the overall kinetic energy to increase. At intermediate wavenumbers the energy spectrum undergoes a transition from a limited $k^{-5 / 3}$ inertial range to $k^{-2}$ scaling recently predicted in the literature. Although the Reynolds stress tensor remains approximately isotropic and threecomponent, evidence for anisotropy and quasi- two-dimensionality in length scales and spectra in different velocity components and directions is strong. The small scales are found to deviate from local isotropy, primarily as a result of anisotropic transfer to the high wavenumbers. To understand the spectral dynamics of this flow we study the detailed behavior of nonlinear triadic interactions in wavenumber space. Spectral transfer in the velocity component parallel to the axis of rotation is qualitatively similar to that in nonrotating turbulence; however the perpendicular component is characterized by a greatly suppressed energy cascade at high wavenumbers and a local reverse transfer at the largest scales. The broader implications of this work are briefly addressed.
\end{abstract}

Key words. direct numerical simulations, rotating turbulence, anisotropy and quasi- two-dimensionality

Subject classification. Fluid Mechanics

1. Introduction. Turbulent flows subjected to solid-body rotation occur in many enginecring and geophysical applications, such as in turbomachinery with rotating blades, and in problems involving the rotation of the Earth. The effects of rotation (through a Coriolis force) on turbulence structure are known to be subtle, yet profound: although it has no direct role in the kinetic energy budget, rotation weakens the fundamental property of an energy cascade from the large scales to the small scales (e.g., Bardina et al. [1], Mansour et al. [2]). However, as pointed out by Speziale [3] most classical turbulence models have been developed without accounting for Coriolis forces and consequently they perform poorly in rotating flows which require the use of non-inertial frames of reference. Recognition of these limitations has provided much motivation for recent work (e.g., Refs. 4-7) aimed at incorporating rotation effects in modeling.

From the viewpoint of physical understanding (and modeling at a fundamental level), a most important test case is that of initially homogeneous isotropic turbulence subjected to uniform rotation, free of other complicating effects. Because of difficulties (cited by Jacquin et al. [8]) in achieving these conditions in experiments (e.g. Wigeland \& Nagib [9]), numerical simulation is an attractive approach (Speziale \& So [10]). Both direct and large-eddy simulations (DNS and LES), supported by experimental data (Jacquin et al. [8]), demonstrate that demonstrate that $[1,11]$ as a result of reduced energy transfer, strong rotation also leads to

${ }^{*}$ School of Aerospace Engineering, Georgia Institute of Technology, Atlanta, GA 30332. This research was supported in part by NSF Grant No. CTS-9307973.

$\dagger$ Institute for Computer Applications in Science and Engineering, NASA Langley Research Center, Hampton, VA 23681 and IBM Research Division, T.J. Watson Research Center, P.O. Box, 218, Yorktown Heights, NY 10598. This research was supported by the National Aeronautics and Space Administration under NASA Contract Nos. NAS1-19480 and NAS1-97046 while the second author was in residence at the Institute for Computer Applications in Science and Engineering (ICASE), NASA Langley Research Center, Hampton, VA 23681. 
reduced cnergy dissipation rates. On the other hand, the dynamics of the resulting anisotropy development is less well established. Most of the available data indicate that the Reynolds stress tensor $\left(\left\langle u_{i} u_{j}\right\rangle\right)$ remains approximately isotropic: for instance, Speziale et al. [11] and Mansour et al. [2] observed linear isotropic decay by viscosity as the nonlinear energy transfer is greatly reduced. However, anisotropy can still be manifested in greatly increased length scales along the axis of rotation (Jacquin et al. [8], Mansour et al. [2], Cambon et al. [12]). This quasi- two-dimensional state differs from the classical Taylor-Proudman Theorem [13], in which all nonlinear terms are neglected.

It may be noted that most of the numerical simulations cited above were performed for decaying isotropic turbulence at low Reynolds numbers without an inertial range. A common approach to increase the Reynolds number is to supply energy through numerical forcing to compensate for the cffects of viscous decay. At the same time, because the primary effect of rotation is to modify the energy transfer to smaller scales, it is illuminating to examine how turbulence under rotation responds to a source of energy input at the largest scales of the flow. In low-resolution simulations Hossain [14] has found that energy input at intermediate scale sizes can lead to strong-dimensionalization and an inverse cascade to the largest scales. Howcver, to fully describe the nature of the nonlinear energy transfer it is essential to address the detailed behavior of triadic interactions in wavenumber space, which represent elemental couplings within groups of Fourier modes whose wave-vectors form closed triangles (Brasseur \& Corrsin [15]). In the case of non-rotating turbulence, analyses of DNS databases (Domaradzki \& Rogallo [16], Zhou [17]) have provided considerable insights concerning the roles of different scale sizes in the energy transfer process. Naturally, knowledge of these nonlinear scale interactions has implications (Domaradzki \& Liu [18], Zhou \& Vahala [19]) for largeeddy simulations in which the effects of the small scales on the large scales must be modeled, and indeed this has been used to devise a new approach in sub-grid scale modeling (Refs. 20, 21). In contrast, current understanding of spectral transfer in rotating turbulence is much less complete, and no similarly detailed analysis of triadic energy transfer based on DNS is currently known. Yet the value of a spectral approach for rotating turbulence is evident in EDQNM closure theories (Cambon \& Jacquin [22], Cambon et al. [23]).

Our objectives in this paper are to study the response of isotropic turbulence under uniform solid-body rotation in the presence of external energy input at the large scales, and to examine the spectral dynamics underlying this response. Direct numerical simulations with $256^{3}$ grid points are carried out using Fourier pseudo-spectral methods. Initially the Taylor-scale Reynolds number $\left(R_{\lambda}\right)$ is about 140 , which is sufficiently high for a limited $k^{-5 / 3}$ inertial range to exist in the energy spectrum, with a value of the Kolmogorov constant in good agreement with experiment (see Yeung \& Zhou [24]). Subsequently the spectrum is found to undergo a transition to $k^{-2}$ scaling, as predicted by a recent phenomenological theory ([25]). A substantial part of this paper is devoted to a comprehensive description of the development of different anisotropy measures in both physical and spectral space. We attempt to explain the observed behavior through analyses of Reynolds stress budgets and triadic energy transfer, where differences with non-rotating turbulence will be emphasized. In particular, at both low and high wavenumbers we distinguish between energy transfer for velocity components parallel and perpendicular to the axis of rotation.

The remainder of this paper is organized as follows. In Sec. II we briefly review the relevant mathematical background and the numerical method. Results are given in Sec. III, within four subsections which focus respectively on (a) the evolution of kinetic energy and dissipation rate, (b) anisotropy measures, (c) budgets for the Reynolds stress tensor and (d) spectral transfer analyses. In Sec. IV we summarize our results and discuss briefly the possible wider implications of this work. An Appendix addresses the coupling of numerical and physical effects responsible for early-time transients in the DNS data. 
2. Background and numerical method. We consider incompressible turbulence subjected to uniform solid-body rotation at rate $\underline{\Omega}$ but without mean velocity gradients. In general rotation gives rise to both centrifugal and Coriolis forces, but in this case when the motion is viewed in a rotating frame of reference only the Coriolis force is relevant. The fluctuating momentum equation in usual Cartesian tensor notation is

$$
\frac{\partial u_{i}}{\partial t}+u_{j} \frac{\partial u_{i}}{\partial x_{j}}=-\frac{1}{\rho} \frac{\partial p}{\partial x_{i}}-2 \epsilon_{i j k} \Omega_{j} u_{k}+\nu \frac{\partial^{2} u_{i}}{\partial x_{j} \partial x_{j}}+f_{i}
$$

where $\epsilon_{i j k}$ is the alternating symbol and $\underline{f}$ is an additional external force to be specified. Because the initial conditions are isotropic, the orientation of $\underline{\Omega}$ is statistically immaterial; for definiteness we take $\underline{\Omega}=(0,0, \Omega)$.

The effects of rotation on the Reynolds stress and energy spectrum tensors are important in this work. With the use of homogeneity in space, the Reynolds stress budget equation can be written as

$$
\frac{d R_{i j}}{d t}=\Pi_{i j}+W_{i j}-D_{i j}+F_{i j}
$$

where $\Pi_{i j}=(2 / \rho)\left\langle p s_{i j}\right\rangle$ represents the (slow) pressure-strain correlation,

$$
W_{i j}=2 \Omega\left[\epsilon_{i k 3}\left\langle u_{j} u_{k}\right\rangle+\epsilon_{j k 3}\left\langle u_{i} u_{k}\right\rangle\right]
$$

(with the choice of $\underline{\Omega}$ above) is the rotation term, $D_{i j}=2 \nu\left\langle\left(\partial u_{i} / \partial x_{k}\right)\left(\partial u_{j} / \partial x_{k}\right)\right\rangle$ is the dissipation tensor, and $F_{i j}=\left\langle\left(u_{i} f_{j}+u_{j} f_{i}\right)\right\rangle$ is the rate of change due to the external force. Both $\Pi_{i j}$ and $W_{i j}$ are traceless and hence represent important intercomponent transfer processes, although they influence the kinetic energy budget only indirectly. The pressure fluctuation field can be obtained by solving (in pseudo-spectral manner) the Poisson equation

$$
\nabla^{2}\left(\frac{p}{\rho}\right)=-\frac{\partial u_{i}}{\partial x_{j}} \frac{\partial u_{j}}{\partial x_{i}}+2 \Omega \epsilon_{i j 3} \frac{\partial u_{j}}{\partial x_{i}}
$$

For convenience we note here that the only non-trivial components of $W_{i j}$ are:

$$
W_{11}=4 \Omega\left\langle u_{1} u_{2}\right\rangle, \quad W_{22}=-4 \Omega\left\langle u_{1} u_{2}\right\rangle, \quad W_{12} \equiv W_{21}=2 \Omega\left(\left\langle u_{2}^{2}\right\rangle-\left\langle u_{1}^{2}\right\rangle\right)
$$

The rate of change of the kinetic energy $K=\frac{1}{2}\left(u_{i} u_{i}\right)$ is given by

$$
d K / d t=\dot{F}-\epsilon
$$

where $\dot{F} \equiv \frac{1}{2} F_{i i}$ is the forcing energy input rate, and $\epsilon$ is the dissipation rate. As to be shown later, both terms in this equation are strongly influenced by rotation.

With the choice $\underline{\Omega}=(0,0, \Omega)$, the Fourier-space equivalent of Eq. 1 for the transformed velocity at wave-vector $\underline{k}$ can be written as

$$
\left(\frac{\partial}{\partial t}+\nu k^{2}\right) \hat{u}_{i}(\underline{k}, t)-2 P_{i j}(\underline{k}) \epsilon_{j l 3} \Omega \hat{u}_{l}(\underline{k})=-P_{i j}(\underline{k}) \hat{H}_{i}(\underline{k})+\hat{f}_{i}(\underline{k})
$$

where carets denote Fourier coefficients, $H_{i}=\partial\left(u_{i} u_{j}\right) / \partial x_{j}$ is used as a shorthand for the nonlinear term, and the tensor $P_{i j}(\underline{k})=k_{i} k_{j} / k^{2}-\delta_{i j}$ represents projections onto the plane perpendicular to $\underline{k}$ in wavenumber space. The evolution equation for the energy spectrum tensor, defined as $E_{i j}(\underline{k}, t) \equiv \frac{1}{2}\left\langle\hat{u}_{i} \hat{u}_{j}{ }^{*}+\hat{u}_{i}{ }^{*} \hat{u}_{j}\right\rangle$ (where asterisks denote complex conjugates) can be written in a form corresponding to Eq. 2, as

$$
\frac{\partial E_{i j}(\underline{k}, t)}{\partial t}=T_{i j}(\underline{k})+A_{i j}(\underline{k})-D_{i j}(\underline{k})+F_{i j}(\underline{k}),
$$


where $T_{i j}(\underline{k})$ represents encrgy transfer by the nonlinear terms,

$$
A_{i j}(\underline{k})=\Omega \epsilon_{l m 3}\left[P_{j l}(\underline{k}) E_{i m}(\underline{k})+P_{i l}(\underline{k}) E_{j m}(\underline{k})\right]
$$

represents Coriolis terms, $D_{i j}(\underline{k})=2 \nu k^{2} E_{i j}(\underline{k})$ is the dissipation spectrum tensor, and $F_{i j}(\underline{k})=\operatorname{Rc}\left[\left\langle\hat{u}_{i} \hat{f}_{j}{ }^{*}+\right.\right.$ $\left.\left.\hat{u}_{i}{ }^{*} \hat{f}_{j}\right\rangle\right]$ represents the forcing contributions. It is well-known that in homogeneous turbulence $T_{i j}(\underline{k})$ has the property of a vanishing integral over all wavenumbers for every pair of subscripts. On the other hand, $A_{i j}(\underline{k})$ has a non-vanishing integral but zero trace at every wavenumber mode. In other words, whereas $T_{i j}(\underline{k})$ represents spectral transfer between different scales, $A_{i j}(\underline{k})$ represent transfer between different components. Unlike its physical-space counterpart ( $W_{i j}$, see Eq. 5), all three diagonal elements of $A_{i j}(\underline{k})$ are non-trivial, specifically

$$
\begin{aligned}
& A_{11}(\underline{k})=\Omega\left[P_{11}(\underline{k}) E_{12}(\underline{k})-P_{12}(\underline{k}) E_{11}(\underline{k})\right], \\
& A_{22}(\underline{k})=\Omega\left[P_{12}(\underline{k}) E_{22}(\underline{k})-P_{22}(\underline{k}) E_{12}(\underline{k})\right], \\
& A_{33}(\underline{k})=\Omega\left[P_{13}(\underline{k}) E_{23}(\underline{k})-P_{23}(\underline{k}) E_{13}(\underline{k})\right] .
\end{aligned}
$$

In numerical simulations it is usual practice to collect the spectral quantitics appearing in Eq. 8 into spherical shells of radius $k$ (and thickness $\Delta k=1$ ), effectively as functions of the wavenumber magnitude $(k=|\underline{k}|)$. The transfer spectrum $T(k)=\frac{1}{2} T_{i i}(k)$ can be written in full as

$$
T(k)=\sum_{k-\frac{1}{2} \Delta k \leq\left[\underline{k}^{\prime} \mid<k+\frac{1}{2} \Delta k\right.} \operatorname{Im}\left\langle k_{m}^{\prime} P_{i j}\left(\underline{k}^{\prime}\right) \hat{u}_{i}^{*}\left(\underline{k}^{\prime}\right) \int_{\underline{k}^{\prime}=\underline{p}+\underline{q}} \hat{u_{j}}(\underline{p}) \hat{u}_{m}(\underline{q}) d \underline{p}\right\rangle .
$$

Here the convolution integral on the r.h.s. signifies the collection of all possible triads $\left(\underline{k}^{\prime}, \underline{p}, \underline{q}\right)$ that can be formed in wavenumber space with $\underline{k}^{\prime}$ belonging to the designated wavenumber shell. The effects of different scale sizes on the transfer at scale $1 / k$ can be compared in terms of the detailed transfer function $T(k \mid p, q)$ (Domaradzki \& Rogallo [16]), which is the partial sum of triadic terms with one "leg" of the triad in a shell centered on $p$ and the other centered on $q$. Furthermore $T(k \mid p)$ is the sum of all contributions with at least one leg in the prescribed band $p$. These quantities are related by

$$
T(k)=\sum_{p} T(k \mid p)=\sum_{p} \sum_{q} T(k \mid p, q) .
$$

Similar definitions and relations apply to the component transfers (e.g., $T_{11}(k)$ ), which are obtained simply by suppressing the summation over the subscript $i$ in the definition (11).

In this work we solve the governing equation (1) numerically, using a massively parallel implementation (Yeung \& Moseley [26]) of the well-known pseudo-spectral algorithm of Rogallo [27]). The time advance is carried out in wavenumber space, using an explicit second-order Runge-Kutta (predictor-corrector) scheme. The linear viscous term is treated exactly via an integrating factor in the predictor step. Following Mansour et al. [2], the time-advance procedure is modified to take account of numerical stiffness difficulties arising from high-frequency oscillations associated with high rotation rates. Essentially, the simultaneous system (7) (for $i=1,2,3$ ) in Fourier space is solved in a diagonalized form which also allows the linear Coriolis term to be integrated exactly. This is achieved by introducing a pair of auxiliary variables

$$
\xi_{1}=\frac{1}{k_{12}}\left(\iota k_{2} \hat{v}_{1}-\iota k_{1} \hat{v}_{2}-k \hat{v}_{3}\right)
$$




$$
\xi_{-1}=-\frac{1}{k_{12}}\left(\iota k_{2} \hat{v}_{1}-\iota k_{1} \hat{v}_{2}+k \hat{v}_{3}\right)
$$

where $k_{12}=\sqrt{k_{1}^{2}+k_{2}^{2}}, \iota=\sqrt{-1}$, and $\underline{\hat{v}}=\underline{\hat{u}} \exp \left(\nu k^{2} t\right)$. The linear terms in the resulting evolution equations for $\xi_{1}$ and $\xi_{-1}$ are decoupled and hence can be treated with a new integration factor (of the form $\exp \left(\mp 2 \Omega \iota k_{3} t / k\right)$, respectively). For those Fourier modes with $k_{12}=0$ the definitions 13(a,b) are modified to $\zeta_{1}=\hat{v}_{1}-\iota \hat{v}_{2}$ and $\zeta_{-1}=\hat{v}_{1}+\iota \hat{v}_{2}$, with the integrating factor becoming $\exp (\mp 2 \iota \Omega t)$. Predictor estimates of $\underline{\hat{u}}$ are readily recovered from the updated auxiliary variables after each predictor step. Whereas this procedure incurs a a small increase in CPU time per step, it has the important advantage of avoiding the use of an undesirably large number of time steps that would otherwise be needed to resolve the rotation-induced oscillations.

In this paper we choose the external force $f$ to be that in the stochastic forcing scheme of Eswaran \& Pope [28]. In this scheme, forcing is applied only to the lowest two wavenumber shells $(k \leq 2)$, and is known to result in statistical stationarity if rotation were absent. The forcing term has a finite correlation time, which differs from the Gaussian white-noise forcing used by Smith et al. [29]. Because of the inherent stochastic variability of this method of forcing, and because rotation leads to increased relative energy content at the large scales (for which relatively few samples exist in the solution domain), the simulation results display considerable statistical variability. Consequently, to ensure reliable statistics it is necessary to perform ensemble averaging over multiple independent realizations. Our results are taken primarily from an ensemble average of six such $256^{3}$ simulations with statistically independent initial conditions.

3. Results and analysis. We present ensemble-averaged statistics from a scries of direct numerical simulations at two rotation rates. The grid resolution is $256^{3}$, the initial Taylor-scale Reynolds number is about 140 , and the simulation time period is 6.8 initial eddy-turnover times $\left(T_{E, 0}\right)$. A conventional nondimensional measure of the strength of rotation is given by comparing the rotation time scale $(1 / \Omega)$ to the turbulence time scale $(K / \epsilon)$, giving the turbulent Rossby number $R o_{T} \equiv \epsilon /(2 K \Omega)$. Comparison with other time scales representing large-scale and small-scale motions may also be useful, leading to macro- and microRossby numbers (Cambon et al. [23]) respectively. The definitions and initial and final values of of thesc quantities in the simulations are listed in Table I.

Initially the "longitudinal" and "transverse" macro-Rossby numbers $\left(R o_{l}^{L}\right.$ and $\left.R o_{t}^{L}\right)$ are approximately equal, but eventually differ by a ratio of about 1.8 . We also include the non-dimensional parameter $k_{\Omega} \eta$, where $k_{\Omega}=\left(\Omega^{3} / \epsilon\right)^{1 / 2}$ (first introduced by Zeman [30]) is a characteristic wavenumber of rotation. A value of $k_{\Omega} \eta>1$ indicates that rotation effects extend to scales smaller than the Kolmogorov scale $(\eta)$, which suggests (as to be discussed later) the possibility of deviations from classical concepts of Kolmogorov similarity at the small scales.

Because of computational expense, only two realizations at initial $R o_{T}=0.0039$ have been performed, compared to six for the $R o_{T}=0.0195$ case. In the paragraphs below we present results first on the evolution of energy and dissipation rate, including the shape of the spectrum, and then on anisotropy measures in terms of the Reynolds stress tensor, length scales and component energy spectra. Analyses of Reynolds stress budgets in physical space and spectral transfer in wavenumber space are then used to help explain the observed DNS results. Unless noted otherwise (and primarily in Sec. IV.A) the results given below are for the case of $R o_{T}=0.0195$. 
TABLE I

Initial and final values of Rossby numbers and related non-dimensional parameters in the simulations.

$\begin{array}{lcccc}\Omega & 10.775 & 10.775 & 52.75 & 52.75 \\ t / T_{E, 0} & 0 & 5.84 & 0 & 5.84 \\ R o_{T}=\epsilon /(2 K \Omega) & 0.0195 & 0.0038 & 0.0039 & 0.00064 \\ R o_{l}^{L}=u_{3}^{\prime} /\left(2 L_{33,3} \Omega\right) & 0.0667 & 0.0278 & 0.0133 & 0.00742 \\ R o_{t}^{L}=u_{1}^{\prime} /\left(2 L_{11,3} \Omega\right) & 0.0623 & 0.0155 & 0.0125 & 0.00426 \\ R o^{\omega}=1 /\left(2 \tau_{\eta} \Omega\right) & 0.994 & 0.448 & 0.199 & 0.0872 \\ & & & & \\ k_{\Omega} \eta & 0.357 & 1.177 & 3.99 & 13.13\end{array}$

Notes: (1) $L_{3}$ and $u_{3}^{\prime}$ are the longitudinal integral length scale and r.m.s. velocity along the axis of rotation. (2) $\tau_{\eta}=(\nu / \epsilon)^{1 / 2}$ is the Kolmogorov time scale.

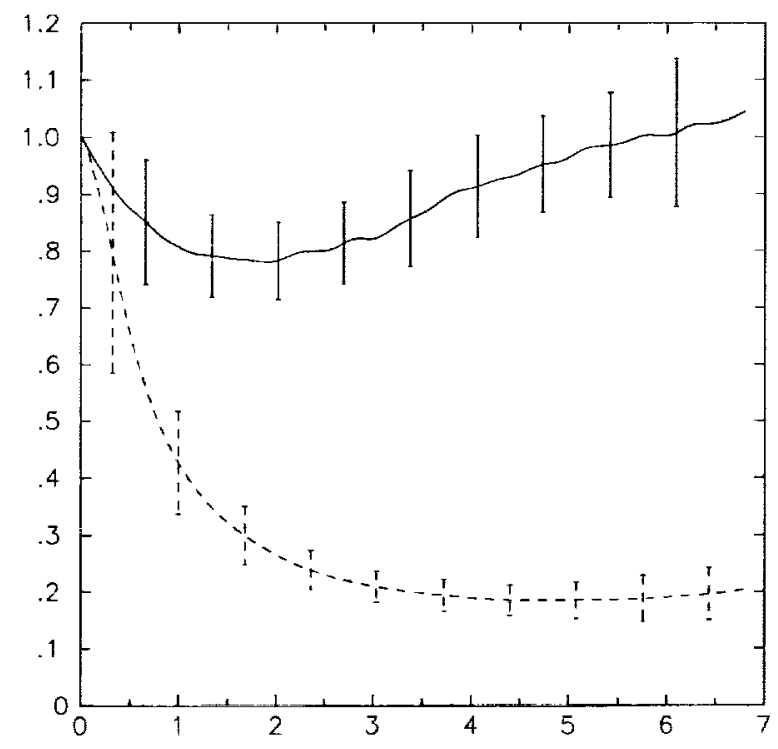

FIG. 1. Evolution of turbulence kinetic energy $\left(K(t) / K_{0}\right.$, solid line) and dissipation rate $\left(\epsilon(t) / \epsilon_{0}\right.$, dashed line) in normalized time $\left(t / T_{E, 0}\right)$ during the simulations, compared with respective initial values. Vertical bars indicate $95 \%$ confidence intervals in an ensemble of six independent realizations. 


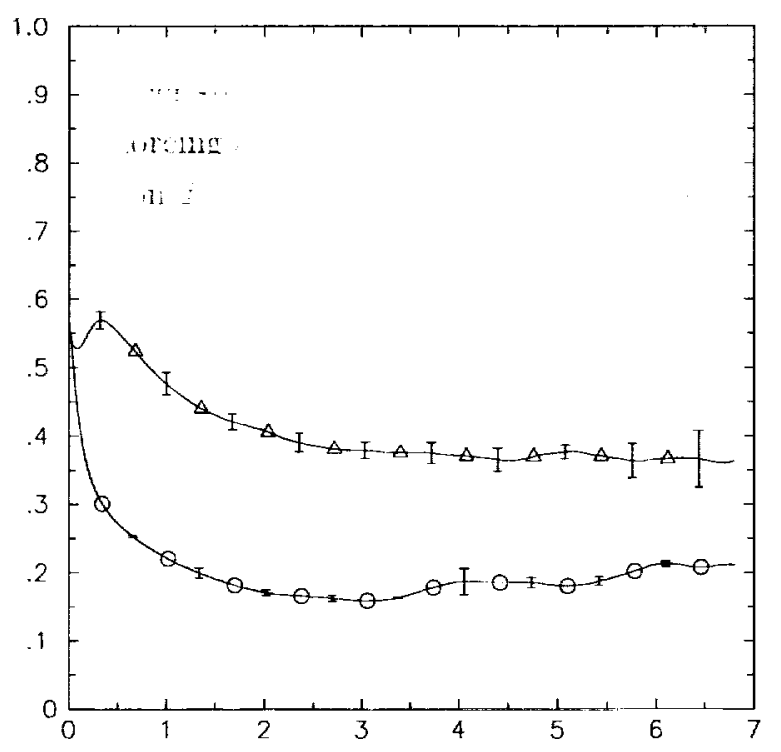

FIG. 2. Evolution of dissipation skeumess $\left(S_{\varepsilon}(t)\right)$ in normalized time $\left(t / T_{E, 0}\right)$ in two series of simulations with $\Omega=10.775$ $(\triangle)$ and $\Omega=52.75(\mathrm{O})$, with confidence intervals shown by the vertical bars.

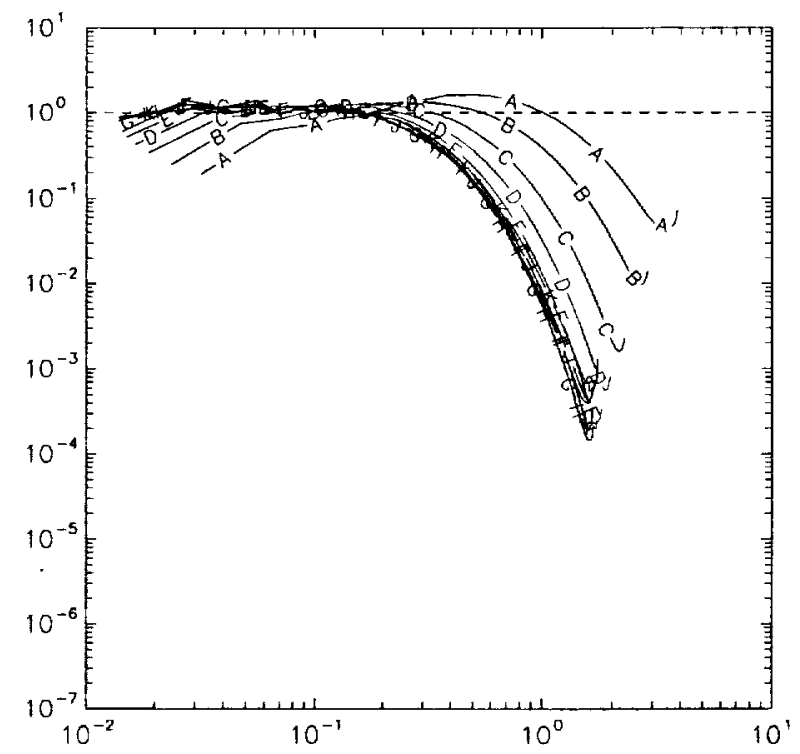

FIG. 3. Energy spectrum normalized by rotation scaling, $\psi(k)=E(k)(\epsilon \Omega)^{1 / 2} k^{-2}$, versus $k / k_{\Omega}$ at different times in the simulations for $\Omega=10.775$. esults are plotted at regular time intervals in increments from $t / T_{E, 0}=0$ to 6.8 (lines $A$ to $\mathrm{K}$ ). Dashed lines are drawn at the value 1.0 for reference.

A. Energy and dissipation. The energy and dissipation rate (which are the subjects of $K-\epsilon$ modeling) provide important global measures of the state of the turbulence under rotation. In Fig. 1 we show the evolution of these quantities normalized by their initial values, for the rotation rate that yields $R o_{T}=0.0195$. Despite the considerable statistical variability (quantified by the confidence intervals shown), it may be clearly seen that the kinetic energy initially decreases somewhat, but subsequently rises (approximately linearly) with time. On the other hand the dissipation rate drops very sharply in the early stages and then remains nearly constant even as the energy begins to rise. In view of the form of the kinetic energy balance (Eq. 6), 


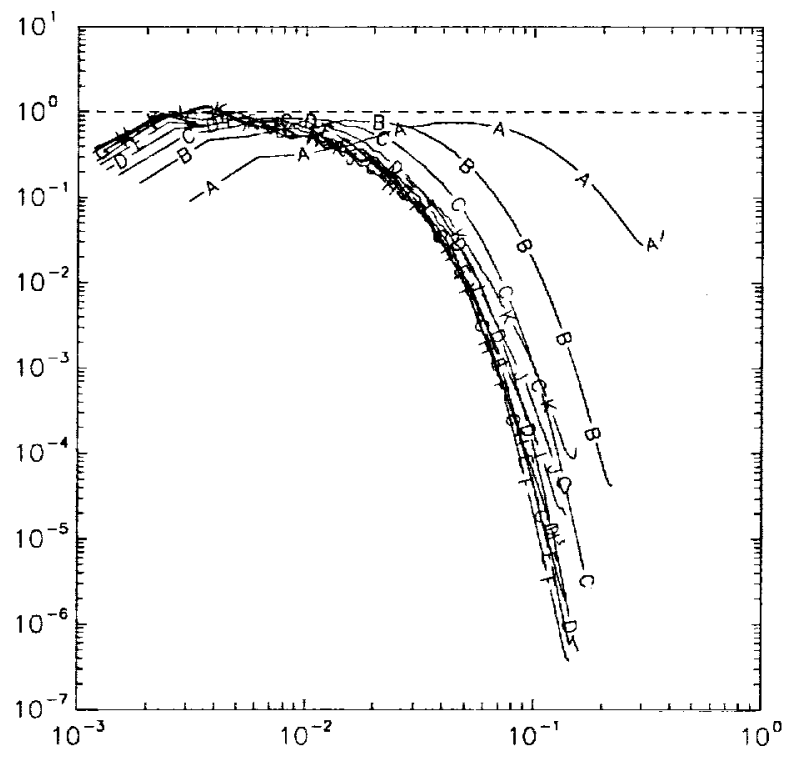

Fic. 4. Same as Fig 3, but for $\Omega=52.75$.
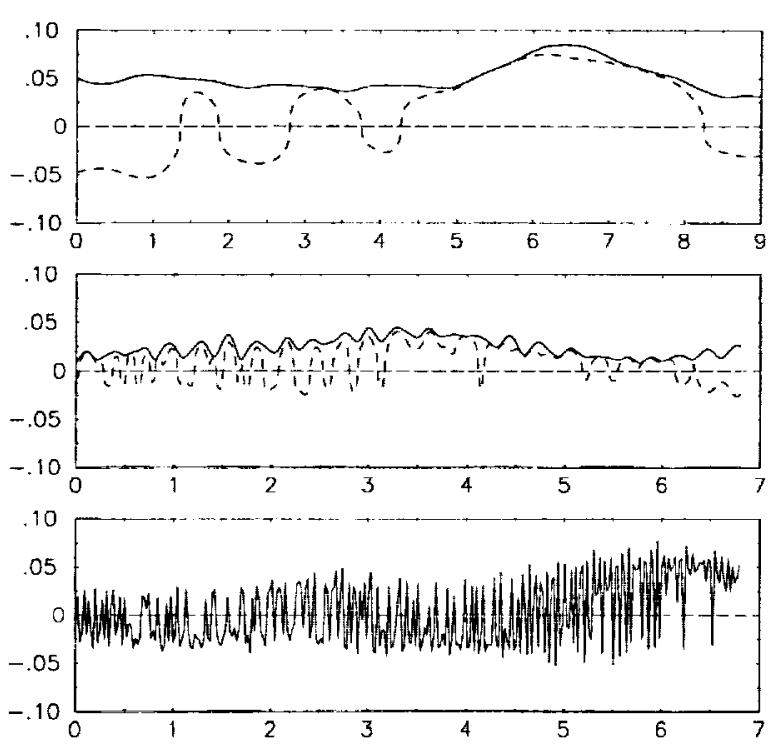

FIG. 5. Evolution of second (solid line) and third (dashed line) principal anisotropy invariants of the Reynolds stress tensor in normalized time $t / T_{E, 0}$, for (from top to bottom) $\Omega=1.0, \Omega=21.55$ and $\Omega=105.5$.

these results suggest that at early times the energy input from forcing is weak, so that the flow is similar to decaying isotropic turbulence in a rotating frame, with a dissipation rate strongly reduced by rotation (Speziale et al. [11]). In this transient period the flow statistics are also subject to a small numerical artifact depending on the manner in which the forcing is initiated. This latter issue is discussed in the Appendix -

The observation of increasing $K$ with near-constancy of $\epsilon$ at later times is of considerable interest. Since in wavenumber space $\epsilon=\int_{0}^{\infty} 2 \nu k^{2} E(k) d k$, this obscrvation implies a change in spectral shape in particular that the energy spectrum is shifted towards lower wavenumbers, together with a reduction in highwavenumber spectral content. A convenient non-dimensional measure of spectral shape is the dissipation 


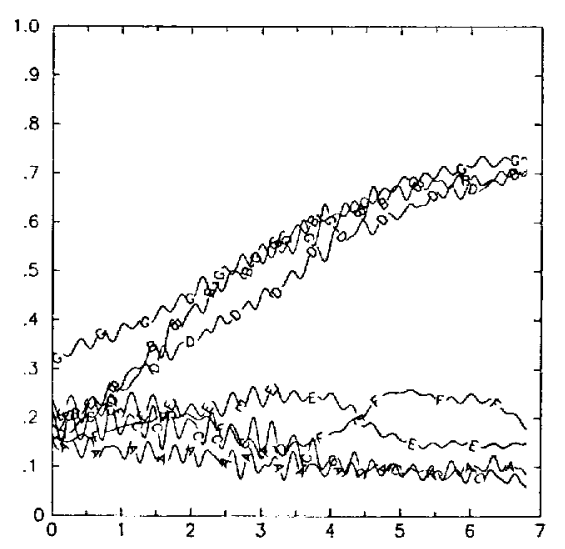

FiG. 6. Evolution of six components of transverse integral length scales $L_{\alpha \alpha, \beta}(\alpha \neq \beta): L_{11,2}(A), L_{11,3}(B), L_{22,1}(C)$, $L_{22,3}(D), L_{33,1}(E)$ and $L_{33,2}(F)$ in normalized time $t / T_{E, 0}$. Evolution of $L_{33,3}$ is indicated by line (G). All are normalized by half of the length $\left(L_{0}\right)$ of the solution domain.

skewness, defined as (from Batchelor [31])

$$
S_{\epsilon}=\frac{4}{35}\left(\frac{15 \nu}{\epsilon}\right)^{3 / 2} \int_{0}^{\infty} \nu k^{4} E(k) d k
$$

(Note: in DNS on an $N^{3}$ grid, integrations in wavenumber space are taken only up to the highest resolvable wavenumber, $k_{\max }=\sqrt{2} N / 3$.) In non-rotating isotropic turbulence $S_{\epsilon}$ is known to be about 0.5 (except at very low Reynolds numbers). Zhou [25] has suggested that at high Reynolds number with an extended inertial range in the spectrum, as rotation rate increases $S_{\epsilon}$ should decrease asymptotically to zero in proportion to $\Omega^{-1 / 4}$.

The evolution of $S_{\epsilon}$ for both rotation rates is shown in Fig. 2. It may be clearly seen that $S_{\epsilon}$ is strongly reduced by rotation, and is characterized by quasi-stationary levels at asymptotically large times. However these "equilibrium" values appear to decrease with rotation rate faster than $\Omega^{-1 / 4}$. This deviation is most likely due to moderate Reynolds numbers in DNS, so that the assumption of an "extended" inertial range is not satisfied. It is also worth noting that the statistical variability in $S_{\epsilon}$ is small, which in turn implies that the functional form of the modified energy spectrum in the simulations is relatively robust.

As suggested by Zhou [25], except at very weak rotation rates the classical picture of Kolmogorov similarity scaling must be modified to include the effects of additional length and time scales due to rotation in the spectral processes. Such departures from classical results are especially pronounced for the high 
rotation casc. In this case the characteristic rotation wavenumber $\left(k_{\Omega}\right)$ is found to be greater than both the Kolmogorov wavenumber $\left(k_{\eta}=1 / \eta\right)$ and the highest resolvable wavenumber $\left(k_{\max }\right)$ in the simulations, and consequently rotation effects extend beyond the viscous cutoff in wavenumber space.

In the rotation-modified inertial range, different theoretical arguments have been used in the literature (Zhou [25] to suggest that for $1 / L \gg k \ll k_{\Omega}$ (where $L$ represents the large scales) the energy spectrum is of the form

$$
E(k)=C_{\Omega}(\epsilon \Omega)^{1 / 2} k^{-2}
$$

where the value of the constant $C_{\Omega}$ (if universal) is yet to be ascertained. In Figs. 3 and 4 we show the development of the normalized spectrum $\psi(k)=E(k)(\epsilon \Omega)^{-1 / 2} k^{2}$ at different times, for both rotation rates in the simulations. Initially $\psi(k)$ exhibits a short scaling range of slope $1 / 3$, corresponding to the presence of a (short) $k^{-5 / 3}$ inertial range prior to rotation. It can be seen that the simulation data provide fairly strong evidence of $k^{-2}$ behavior over a significant range of wavenumbers. If all requirements for a rotation-modified inertial range were met then the height of a plateau in $\psi(k)$ over an appropriate wavenumber range could be used to infer the value of $C_{\Omega}$. In this way our estimates for $C_{\Omega}$ are about 1.2 and 1.0 respectively for $R o_{T}=0.0195$ and 0.0039. Admittedly, the Reynolds number in the simulations may not be high enough for an accurate determination of $C_{\Omega}$. Yet we may note that these values are closer to the phenomenological estimate $C_{K}^{3 / 4} \approx 1.42$ (where $C_{K} \approx 1.6$ is the Kolmogorov constant) by Zhou [25] than higher values proposed by Canuto \& Dubovikov [32].

Because the small scales receive their energy primarily from the large scales, the present observations of reduced dissipation rate and a more negative exponent for the inertial range spectrum are strong indicators of reduced spectral transfer, which is to be studied further in Sec. IV.D.

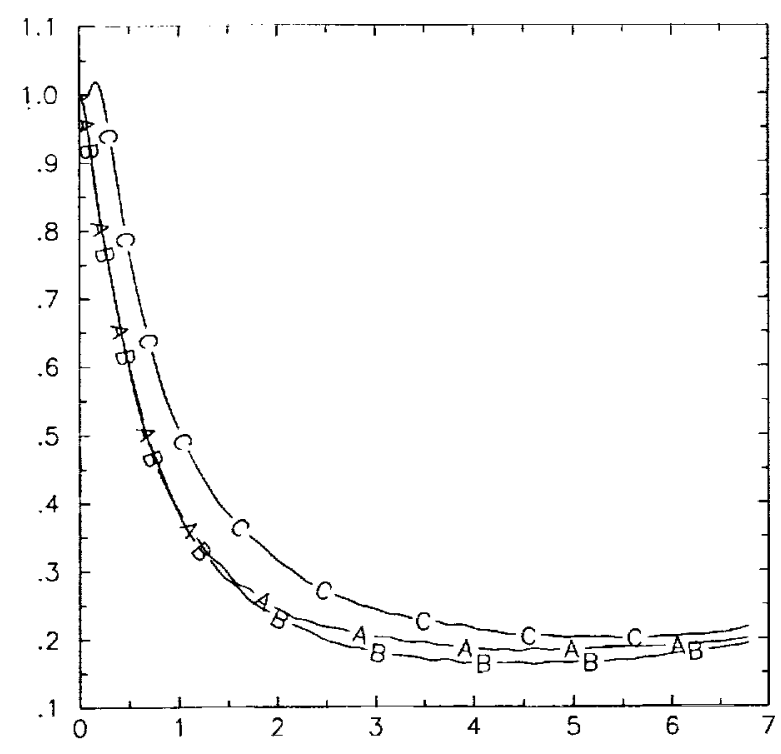

FiG. 7. Evolution of vorticity component variances ( $A, B, C$ for $\omega_{1}, \omega_{2}, \omega_{3}$ respectively), normalized by initial values. Normalized $\left\langle\omega_{1}^{2}\right\rangle,\left\langle\omega_{2}^{2}\right\rangle,\left\langle\omega_{3}^{2}\right\rangle$. 


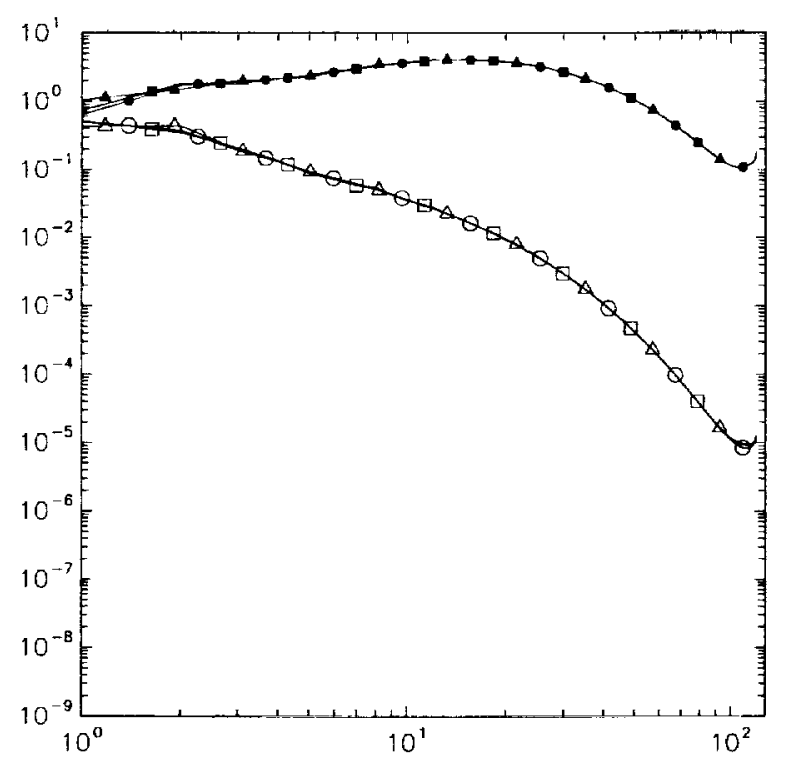

FIG. 8. Energy ( $E_{\alpha \alpha}(k)$, open symbols) and vorticity $\left(V_{\alpha \alpha}(k)\right.$, closed symbols) component spectra at initiation of simulations. Triangles, circles and squares denote $\alpha=1,2,3$ respectively.

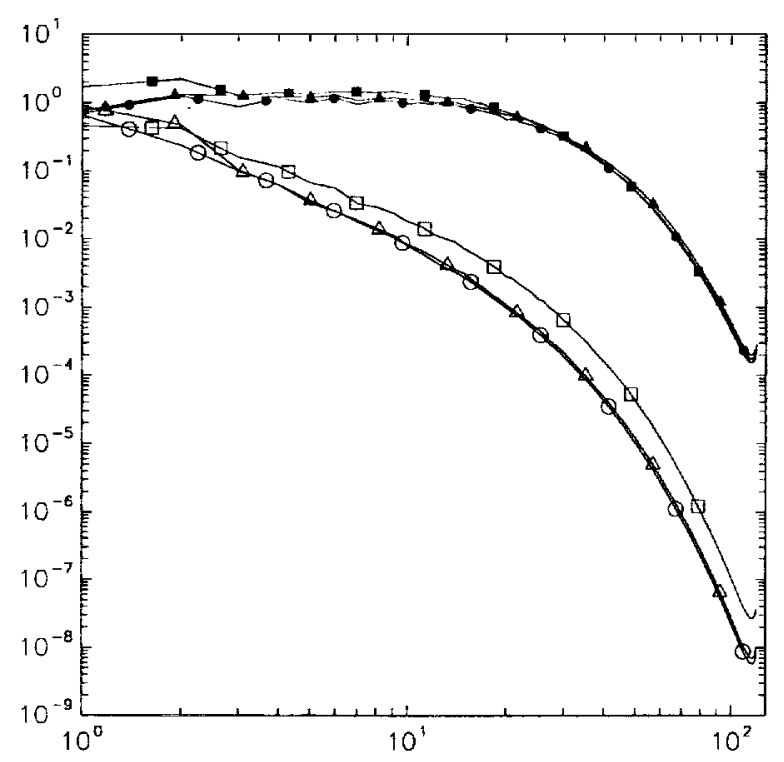

FIG. 9. Same as Fig. 8 , but at at $t / T_{E, 0}=3.4$ (half-way through the simulation period). Triangles, circles and squares denote $\alpha=1,2,3$ respectively.

B. Anisotropy measures. Here we examine the degree to which turbulence becomes anisotropic (or remains isotropic) under the effects of rotation. Both physical- and spectral-space quantities containing length scale information are considered.

In second-order modeling the behavior of the Reynolds stress anisotropy tensor, $b_{i j} \equiv\left\langle u_{i} u_{j}\right\rangle / 2 K-1 / 3 \delta_{i j}$ is very important, especially in terms of its second and third principal invariants (Lumley \& Newman [33]). 


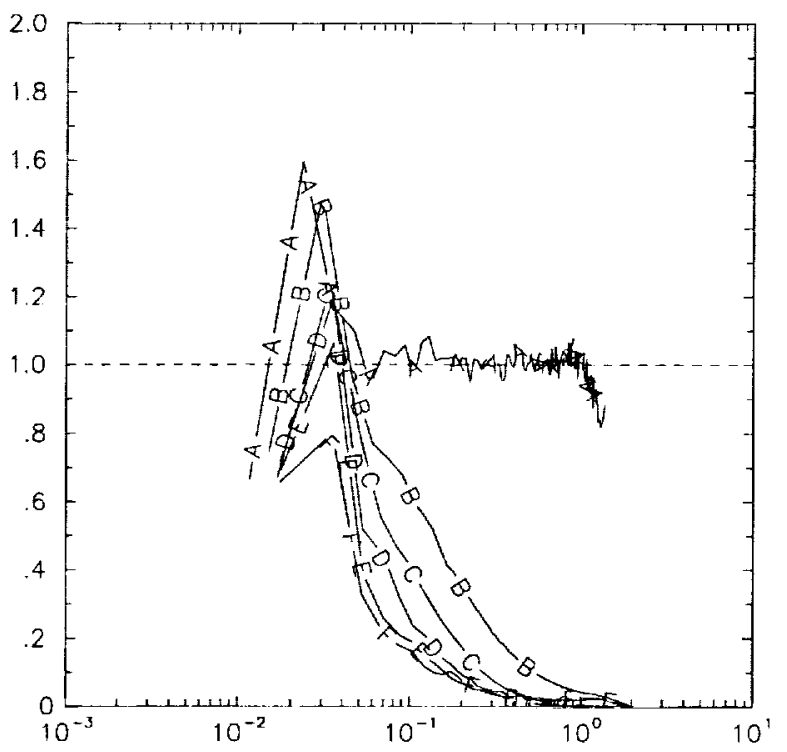

FIG. 10. Spectral isotropy coefficient $\hat{I}_{3}(k)$ verse $k$ (Eq. 18) for velocity component parallel to $\underline{\Omega}$ at regular time intervals $t / T_{E, 0}=0$ to 6.8 .
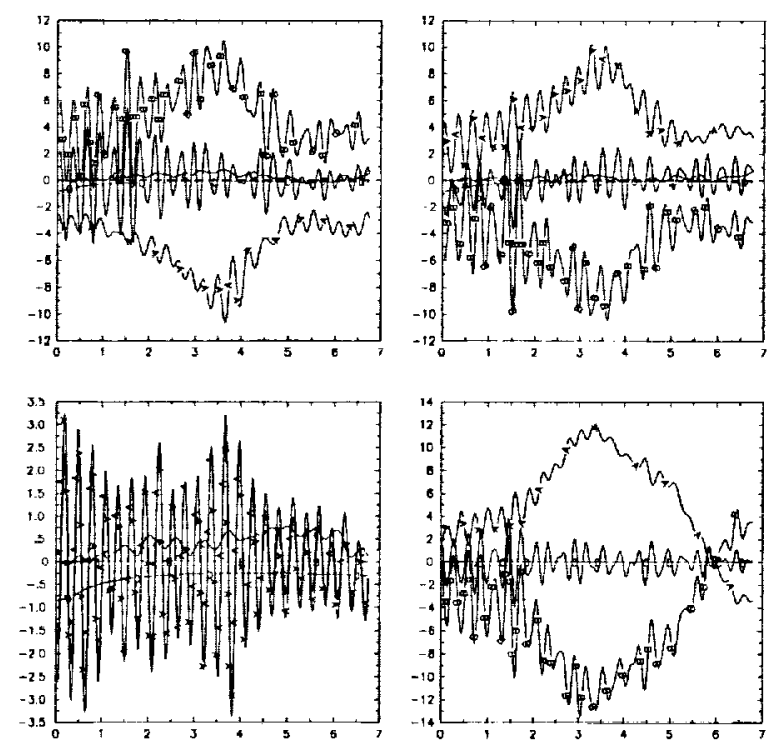

FIG. 11. Evolution of different terms in the Reynolds stress component budgets verse $t / T_{E, 0}:$ (a) $d R_{11} / d t$, (b) $d R_{22} / d t$, (c) $d R_{33} / d t$, and (d) $d R_{12} / d t$. The lines are: $\Pi_{i j}(A), W_{i j}(B), F_{i j}(C), D_{i j}(D)$. The overall rate of change (dR $R_{11} / d t$, etc.) are indicated by unmarked solid lines.

In Fig. 5 the cvolution of these invariants are presented in the form

$$
I_{2}=\left(\frac{1}{6} b_{i j} b_{j i}\right)^{1 / 2} ; \quad I_{3}=\left(\frac{1}{6} b_{i j} b_{j k} b_{k i}\right)^{1 / 3}
$$

The numerical factors and exponents here are chosen such that both $I_{2}$ and $I_{3}$ are equal to $1 / 3$ in the hypothetical limit of one-dimensional turbulence; $I_{2}$ is a non-negative measure of the degree of anisotropy, whereas the sign of $I_{3}$ indicates whether the $R_{i j}$ has one or two dominant eigenvalues. It should be noted that even in nominally isotropic turbulence some anisotropy is expected for spatially-averaged statistics at 


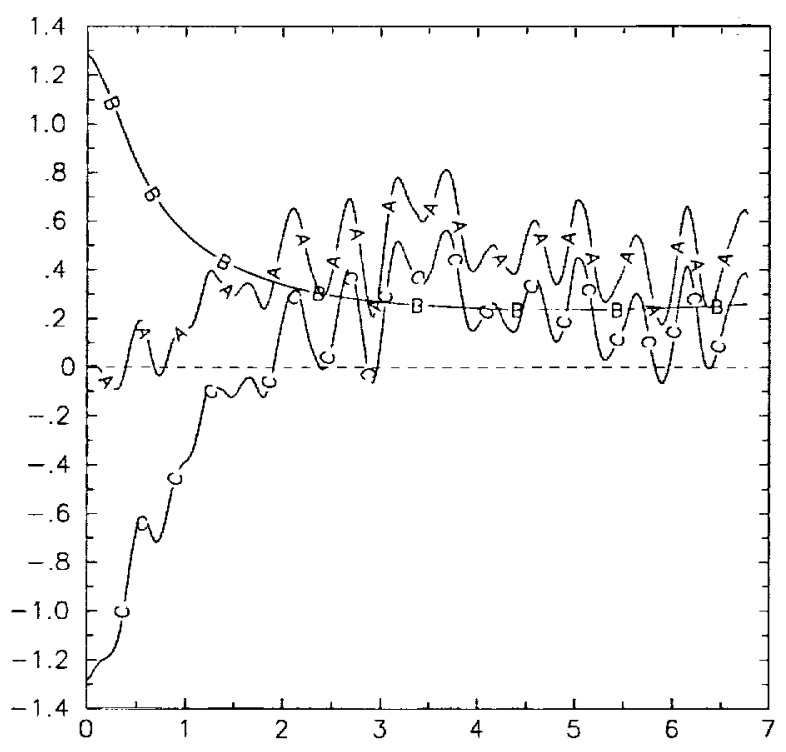

FIG. 12. Evolution of the turbulence kinetic energy balance verse $t / T_{E, 0}: \frac{1}{2} F_{i i}(A),\langle\epsilon\rangle(B)$, and $d K / d t(C$, as the difference $A-B$ ).

instantaneous time. A striking effect of rotation on $I_{3}$ is to cause rapid oscillations in sign which vary according to the rotation time scale $1 / \Omega$ (and are indicative of phase-scrambling effects [34]. Yet the anisotropy levels in this figure under rotation are not appreciably greater than those found in (nominally) isotropic turbulence without rotation. Consequently we may infer that the Reynolds stress tensor indeed remains, at least to a good approximation, isotropic and three-component. However, because componentiality and dimensionality are not equivalent concepts (Reynolds \& Kassinos [35]), this finding does not preclude the development of quasi- two-dimensional characteristics.

The issue of quasi- two-dimensionality can be addressed by a comparison of length scales in different coordinate directions. Figure 6 shows the evolution of the transverse integral length scales, defined as (with no sum over Greek subscripts)

$$
L_{\alpha \alpha, \beta}=\frac{1}{\left\langle u_{\alpha} u_{\alpha}\right\rangle} \int_{0}^{\infty}\left\langle u_{\alpha}(\underline{x}) u_{\alpha}\left(\underline{x}+r \underline{e}_{\beta}\right)\right\rangle d r,
$$

in terms of the two-point correlations along the direction of the unit vector $\underline{e}_{\beta}$ (with $\alpha \neq \beta$ ). The reasons for temporal oscillations are the same as in Fig. 5. In isotropic turbulence all six components (as $\alpha$ ranges from 1 to 3 , with $\alpha \neq \beta$ ) are statistically identical. The present data shows, however, that rotation causes the length scales along the axis of rotation (namely $L_{11,3}$ and $L_{22,3}$ ) to grow steadily over time, becoming much larger than the others. On the other hand, $L_{11,2}$ and $L_{22,1}$ representing motions in the plane normal to the rotation axis $(\underline{\Omega})$ are relatively small, whereas the length scales $L_{33,1}$ and $L_{33,2}$ of the velocity component aligned with $\underline{\Omega}$ are about the same as prior to rotation. These observations suggest that the large scale motions are lengthened along the axis of rotation but more compact within the plane orthogonal to this axis. We note also that the longitudinal integral scale, $L_{33,3}$ along the axis of rotation also increases with time, and that longitudinal and transverse scales in this direction are all approximately equal at later times.

There may be some concern that as the integral length scales $L_{11,3}, L_{22,3}$, and $L_{33,3}$ grow to values comparable to the length of the solution domain, the simulation results may be influenced by the use of 
periodic boundary conditions (Squires et al. [36]). In this paper our strategy is to limit these effects by restricting the length of the simulation time period. Nevertheless, it is clear (e.g., Figs. 1, 2, 5) that systematic trends are already well established at times before this numerical effect becomes a significant issue.

A natural question that arises here is whether the large-scale motions arc associated with vorticity in the direction of $\underline{\Omega}$. Such a scenario is evidently consistent with the evolution of component vorticity variances, shown in Fig. 7. It is clear that vorticity fluctuations are preferentially aligned with the axis of rotation, although less so towards the end of the simulations. Visualization results by Kimura \& Herring [37] also indicate that rotation tends to create "tube-shaped" vortices.

To investigate anisotropy properties at different scale sizes we show in Figs. 8-9 the radial spectra $E_{\alpha \alpha}(k)$ and $V_{\alpha \alpha}(k)$ of the component energy and vorticity variances respectively, at the beginning and half-way point of the simulation time period. Consistent with the decrease of dissipation shown in Fig. 1, it may be seen that at high wavenumbers all components of $E_{\alpha \alpha}(k)$ and $V_{\alpha \alpha}(k)$ are greatly reduced by rotation. Results at low wavenumbers are subject to uncertainties due to both statistical sampling and temporal oscillations induced by rotation. Nevertheless, it is clear that the anisotropy characteristics of $E_{\alpha \alpha}(k)$ and $V_{\alpha \alpha}(k)$ differ significantly. We find that $E_{\alpha \alpha}(k)$ is approximately isotropic in the coordinate components at low wavenumbers, but clearly anisotropic and dominated by the $u_{3}$ component at high wavenumbers. On the other hand, $V_{\alpha \alpha}(k)$ is strongly anisotropic and dominated by its spanwise component at low wavenumbers, and yet close to isotropic at high wavenumbers. A remarkable feature of this behavior is that whereas the energy spectrum suggests possible deviations from the concept of local isotropy, this is not reflected in the vorticity spectrum. Because single-point variances of both the velocity and the vorticity are dominated by contributions from the low wavenumber parts of the spectrum, the differences seen here also explain the apparent isotropy of the Reynolds stress tensor (Fig. 6) versus the strong anisotropy of the vorticity variances (Figs. 8 and 9 ).

Another test of isotropy in spectral space is to check whether the longitudinal and transverse components of the one-dimensional spectra satisfy relations expected of isotropic turbulence. For each velocity component $u_{\alpha}$, we shall denote by $E_{\alpha \alpha}^{L}(k)$ and $E_{\alpha \alpha}^{T}(k)$ respectively its one-dimensional spectra with wave-vectors taken in directions longitudinal and transverse to the velocity component itself. At a given wavenumber if isotropy strictly prevails then the "isotropy coefficients"

$$
\hat{I_{\alpha}}(k)=\frac{E_{\alpha \alpha}^{L}(k)-k d E_{\alpha \alpha}^{L}(k) / d k}{2 E_{\alpha \alpha}^{T}(k)}
$$

should be equal to unity. Figure 10 shows the data for $\hat{I}_{3}(k)$ at different times, with component averaging in the $x-y$ plane taken for the transverse spectrum $E_{33}^{T}(k)$. In the case of isotropic turbulence deviations can arise at low wavenumbers because of sampling and limitations and the lack of perfect isotropy of the solution domain (Yeung \& Zhou [24]), and also at the highest few wavenumber bands because of imperfect resolution and incomplete removal of aliasing errors. The remarkable feature in this figure, however, is the fact that rotation causes a pronounced drop of $\hat{I}_{3}(k)$ in all but the lowest few wavenumber bands. This result is found to be due to $E_{33}^{L}(k)$ taking on a much stecper and negative slope than $E_{33}^{T}(k)$ at high wavenumbers. Similarly significant, albeit less dramatic, departures from isotropy have been found in the data for other velocity components through $\hat{I}_{1}(k)$ and $\hat{I}_{2}(k)$ (not shown). 
C. Reynolds stress budgets. To provide some insights into the dynamics of the anisotropy (or lack of) properties of the Reynolds stress tensor, we present here an analysis of the component Reynolds stress budgets, as expressed in Eq. 2. Because of the temporal oscillations duc to rotation (apparent in Figs. 5 and 6) time-resolved results are required in this work. The usual way to calculate Reynolds stress budgets in DNS is to save velocity fields at regular time intervals, and by solving a Poisson equation extract statistics of pressure and other quantities in a post-processing manner. However, this approach is not suitable here, because it would require the archival of an impractically large number of time-dependent three-dimensional datasets. Instead, we use Eq. 2 to calculate the pressure-strain correlation indirectly. The quantitics $R_{i j}$, $W_{i j}, D_{i j}$ and $F_{i j}$ are calculated (conveniently) within the simulations at output time intervals separated by less than one Kolmogorov scalc. This small interval size allows the time derivative $d R_{i j} / d t$ to be obtained accurately by a fourth-order finite-difference scheme, and this is then used to recover $\Pi_{i j}$ from a balance of the other terms in Eq. 2.

Figure 11(a-d) shows the evolution of the budgets of the Reynolds stress tensor for its four nontrivial elements, namely $R_{11}, R_{22}, R_{33}$ and $R_{12}$. The most prominent feature is that the pressure-strain ( $\Pi_{i j}$ ) and rotational $\left(W_{i j}\right)$ contributions are relatively large in magnitude but tend to cancel each other via opposite signs. Physically, this indicates anisotropizing effects due to rotation about a specified coordinate axis are strongly opposed by the isotropizing effects of pressure fluctuations, which are able to re-distribute energy among the different coordinate components efficiently. Contributions from forcing and dissipation appear to be relatively small in magnitude for the normal stresses (equivalent to component energies), and essentially zero for the shear stress $R_{12}$. The net result of the balances is that the rates of change oscillate rapidly in time, but deviate only weakly from zero when measured over a time period of the order of several large-eddy time scales. The lack of a sustained change in $R_{12}$ from zero (starting from isotropic initial conditions) is also consistent with the approximate isotropy between the component energies $\left\langle u_{1}^{2}\right\rangle$ and $\left\langle u_{2}^{2}\right\rangle$ as implied by the results of Fig. 5.

The turbulence kinetic energy balance can be readily examined by taking half of the trace of the results of Fig. 11(a-c), and this is shown in Fig. 12. Because the tensors $\Pi_{i j}$ and $W_{i j}$ are both traceless, only the forcing and dissipation terms remain. Initially, the forcing energy input is essentially zero, since the stochastic process in the forcing scheme is initialized with a random number seed that is independent of the velocity field. Consequently, in the initial period the turbulence decays, although this decay rate is quickly reduced because of a rapid decrease in dissipation. This transient period ends when the forcing energy input attains a quasi-steady state (with strong fluctuations in time) with generally positive values, whereas the dissipation approaches a virtually steady asymptotic value. The net result is a quasi-constant positive rate of change, which explains the approximately linear growth of the kinetic energy seen in Fig. 1.

D. Spectral dynamics and energy transfer. As stated in Sec. I, the modification of nonlinear spectral energy transfer is a major physical aspect of rotating turbulence. Here we examine this process in some detail, including transfers in each velocity component and the roles of different scale sizes.

To provide the basis for comparisons we first show, in Fig. 13, the decomposition $T(k)=\sum T(k \mid p)$ (see Eq. 12) at time $t=0$ before rotation. The curves shown are broadly similar in appearance to established results $[16,38]$ for non-rotating turbulence. The variation of $T(k)$ (the dashed line) with wavenumber indicates 
that the large scales lose energy actively, and that energy is transferred to smaller scales in a cascade reaching into the highest octave in wavenumber space. The intensity of energy loss from the large scales is amplified here because of energy input from forcing at these scales. At high wavenumbers the cascade character of the transfer is manifested in the function $T(k \mid p)$ showing a sequence of successive peaks of opposite signs. For instance, curve $\mathrm{E}$ shows that interactions involving at least one Fourier mode in the range $16 \leq p<32$ tends to remove energy from modes with wavenumber $k$ lower than the mid-point of the $[16,32]$ interval, but to add energy to wavenumbers higher than this threshold. The relative sharpness of both positive and negative peaks also imply that modes differing widely in scale size exchange energy only weakly. Furthermore, by considering geometric constraints (based on the triangle inequality) in wavenumber space as well as the detailed triadic decomposition $T(k \mid p)=\sum_{q} T(k \mid p, q)$ it can be established that energy transfer at high wavenumbers is dominated by nonlocal triadic interactions involving the coupling of two high-wavenumber modes via a low-wavenumber mode.

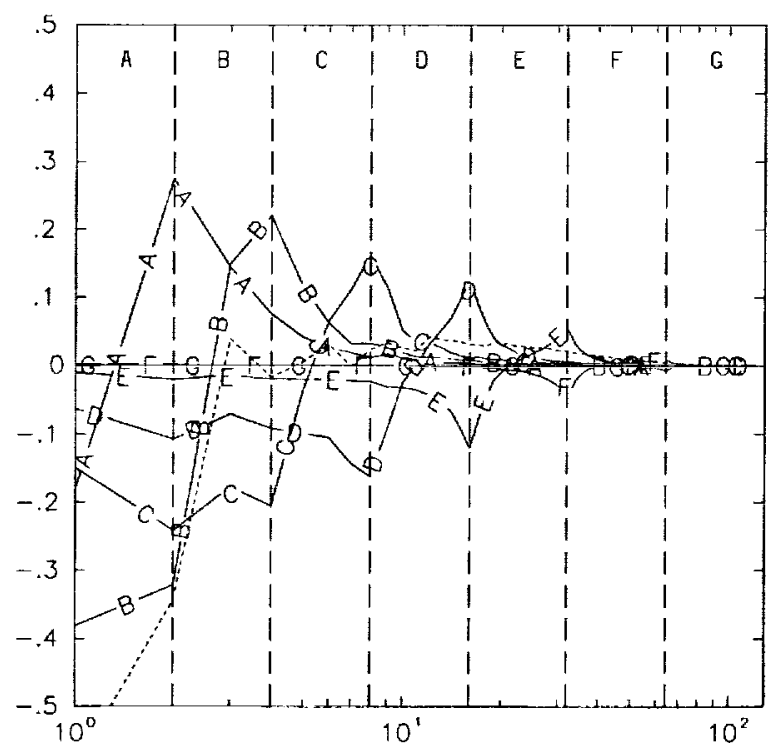

FIG. 13. Energy transfer spectrum $T(k)$ (dashed line) and its decomposition into contributions $T(k \mid p)$ from different wavenumber ranges, for non-rotating isotropic turbulence. The letter symbols represent modes $p$ in octaves identified in top part of the figure.

A contrast is apparent in Fig. 14 which shows energy transfer under rotation at the end of the simulations $\left(t / T_{E, 0}=6.8\right)$, plotted on the same scales as $t=0$ results in Fig. 13. Although the kinetic energy levels at these two time instants are almost the same (see Fig. 1), it is clearly seen that the magnitude of energy transfer activity, in both $T(k)$ and the $T(k \mid p)$ 's, is greatly reduced. The large scales now lose only a small amount of energy, and the energy cascade is hardly felt beyond about $k=32$. The effect of the largest scales on the intermediate scales (in a logarithmic sense) has also become almost minimal: for example, lines $\mathrm{A}$ and $\mathrm{D}$ now indicate that interactions involving modes in the ranges $1 \leq p<2$ and $8 \leq p<16$ together no longer contributes significantly to the net transfer to or from modes $k$ in the other range.

The observation of approximate isotropy in the energy spectrum at low wavenumbers but not the high wavenumbers (see Fig. 9) suggests that the spectral transfer transfer towards the smaller scales under rotation may be significantly anisotropic. This anisotropy is indeed confirmed in Figs.15 and 16 which shows the 


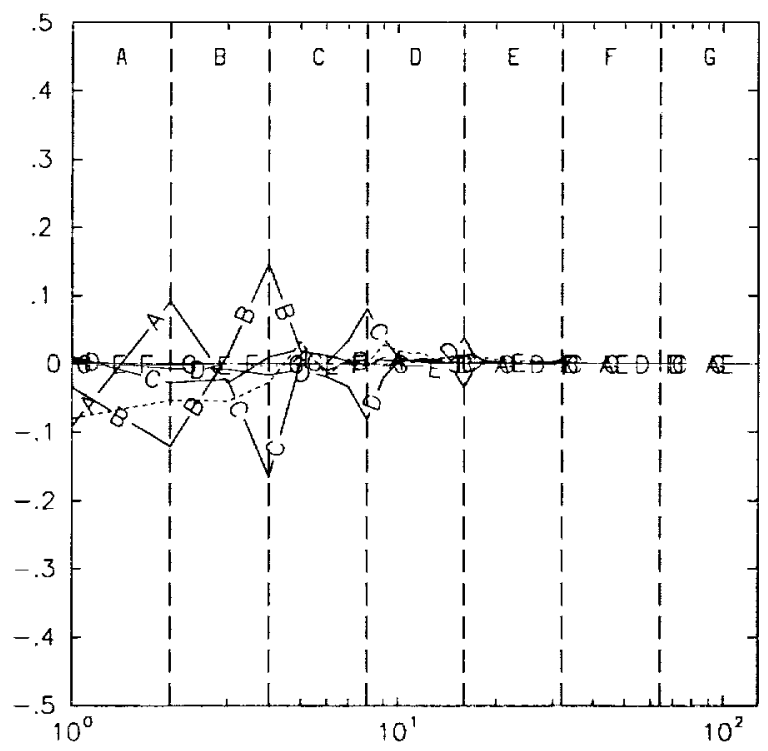

FIG. 14. Same as Fig. 11, but at the end $\left(t / T_{E, 0}=6.8\right)$ of simulation with rotation.

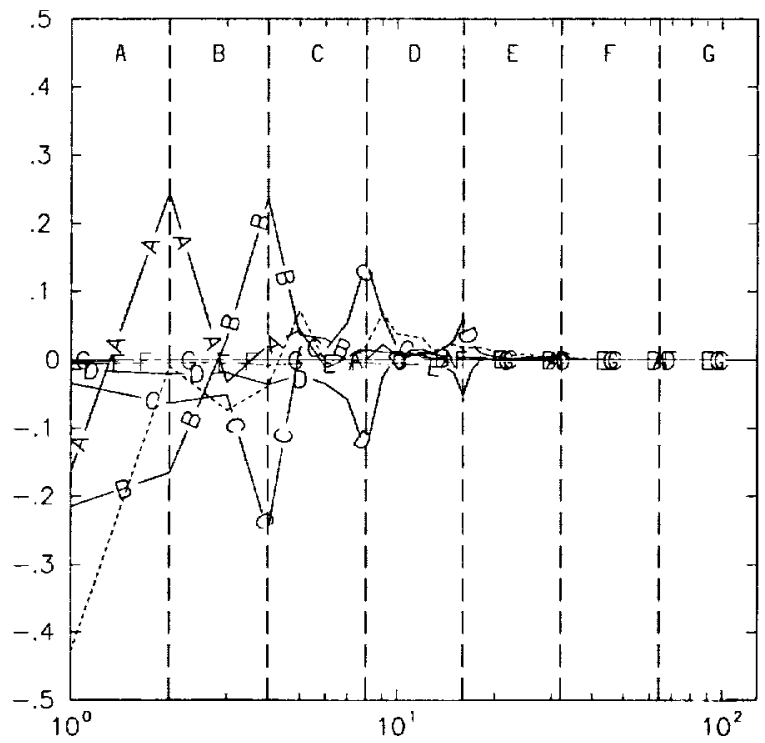

FIG. 15. The decomposition $T_{\alpha \alpha}(k)=\sum_{p} T_{\alpha \alpha}(k \mid p)$ for component transfers in rotating turbulence, for velocity component parallel to $\Omega(\alpha=3)$ Dataset and symbols are the same as in Fig.14

transfers respectively for the velocity components parallel $\left(u_{3}\right)$ and perpendicular $\left(u_{1}\right.$ and $u_{2}$, with component averaging) to the axis of rotation. It may be seen that energy transfer in the parallel component is by far the strongest, and that it retains some of the forward cascade characteristics of energy transfer in non-rotating turbulence. On the other hand, at high wavenumbers the energy cascade for the perpendicular components is virtually shut off. Consequently, the energy that is transferred towards higher wavenumbers resides primarily in the parallel component; this may be regarded as providing an explanation for the anisotropy in the highwavenumber energy spectrum seen in Fig. 9.

Besides a weakened cascade, the energy transfer in the perpendicular components has another important 


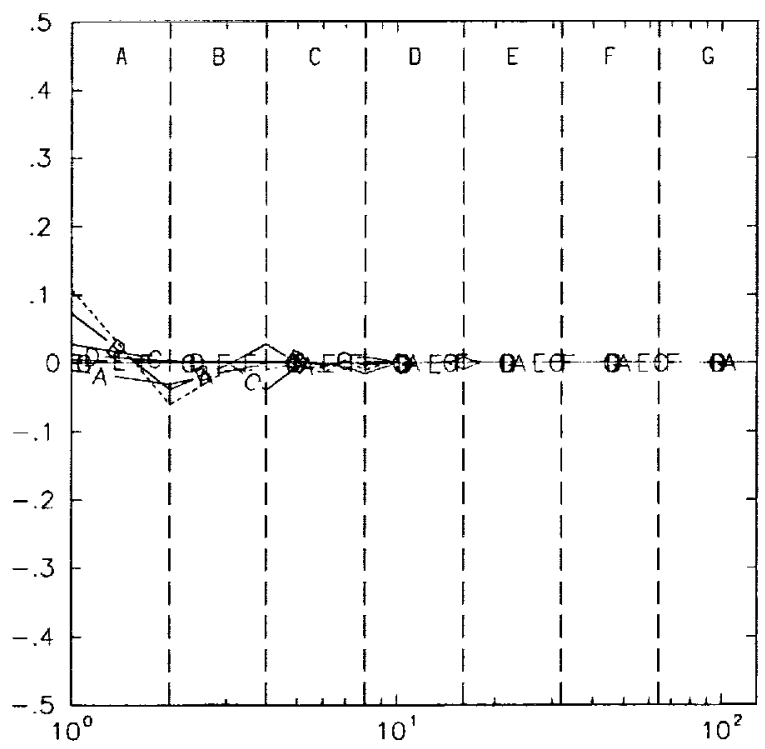

FiG. 16. As as Fig. 15, but for velocity component perpendicular to $\underline{\Omega}$ (average of $\alpha=1,2$ ).

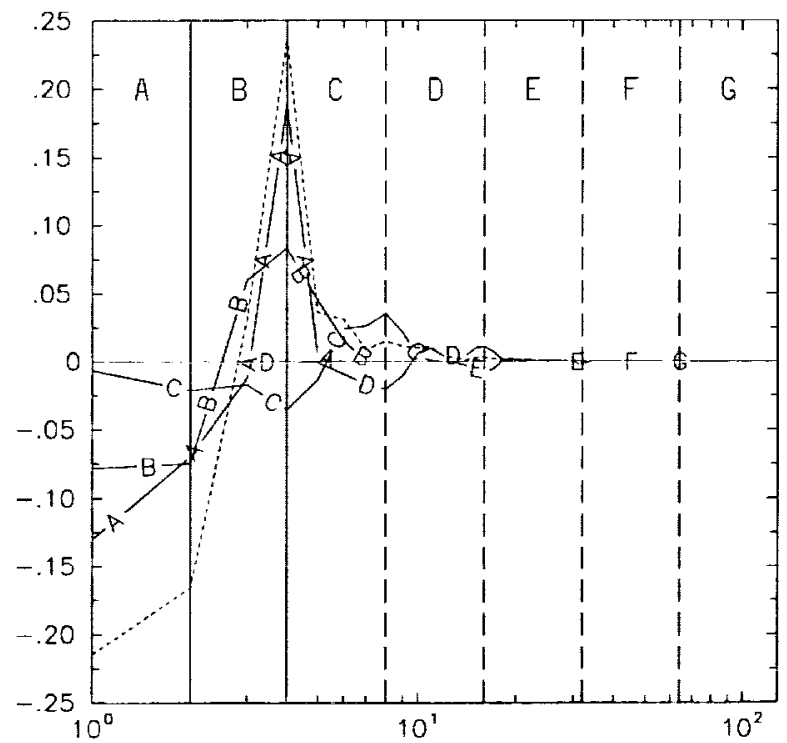

Fig. 17. The detailed component transfer decomposition $T_{\alpha \alpha}(k \mid p)=\sum_{q} T_{\alpha \alpha}(k \mid p, q)$ in the parallel velocity component $(\alpha=3)$ in rotating turbulence, for $2 \leq p<4$ Dashed lines denote $T_{\alpha \alpha}(k \mid p)$, and letter symbols represent modes $q$ in octaves identified in top part of the figure.

characteristic. In Fig. 16 it may be seen that the net transfer (dashed line) for the lowest wavenumber shell $(1 \leq k<1.5$, plotted at $k=1)$ is positive, with the largest contributions coming from the range $2 \leq p<4$ (line B). This is a clear indication of a reverse energy transfer, or inverse cascade, which tends to carry energy towards lower wavenumbers in the spectrum. Because the triangle inequality requires that the remaining leg of a triad with two short legs must also be short, this reverse transfer is apparently the result of local interactions among the large scales.

For a more detailed comparison of the component energy transfers we examine the triadic decomposition 


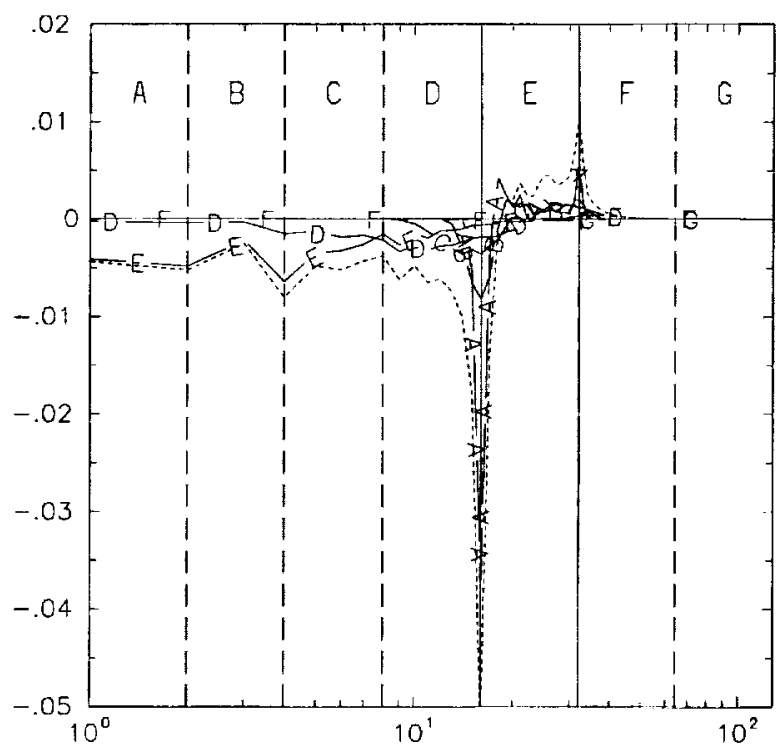

FIG. 18. $16 \leq p<32$.

$T(k \mid p)=\sum_{q} T(k \mid p, q)$ in component form for selected ranges of $p$. To illustrate the typical results we consider, for velocity components parallel (Figs. 17 and 18) and perpendicular (Fig. 19) to $\underline{\Omega}$, this decomposition for (a) the low-wavenumber range $2 \leq p<4$ which contributes most to the inverse cascade effects above, and (b) the higher wavenumber range $16 \leq p<32$ where the cascade to the small scales is of primary interest. The different plotting scales used in each sub-figure reflect the difference in magnitudes for the data in each case. In Figs. 17 and 18 it may be seen from the sign of $T(k \mid p, q)$ that the parallel component displays typical forward transfer behavior, with energy removed from lower wavenumber shells (if $k<p, q$ ) and deposited in higher wavenumber shells (if $k>p, q$ ). In particular (Fig. 18) it is evident that at high wavenumbers nonlocal interactions (line $\mathrm{A}$, in the range with $16 \leq k, p<32$ but $1 \leq q<2$ ) contribute the most to the energy cascade. On the other hand, Figure 19 indicates that the perpendicular velocity modes in the lowest wavenumber shell (centered on $k=1$ ) receives the greatest amount of reverse transfer from triads with both of the other legs in the range $2 \leq p, q<4$ (line B). Furthermore, as seen in Fig. 20 this reverse transfer property is shared by nonlocal interactions with $1 \leq k<2$ but $p$ and $q$ in higher-wavenumber ranges $(16 \leq p, q<32$, line E) as well. Yet these nonlocal contributions are apparently much weaker in magnitude than the local interactions suggested by Fig. 19.

The emphasis of the present discussion has been on the anisotropic aspects of energy transfer under rotation. Another aspect of interest concerns the degree of locality or nonlocality of the transfer, in terms of the range of scales that contribute to a dominant class of the scale interactions. It has been noted in previous literature on non-rotating turbulence (Zhou [17], Zhou et al. [39]) that this latter issue is best addressed by classifying the triadic contributions by a single scale disparity parameter $(s)$ defined to be the ratio between the longest side and the shortest side of each triad. The resulting quantity, $T(k \mid s)$, is very difficult to calculate (Yeung [40]) but can be estimated from the $T(k \mid p, q)$ data collected over octave bands (as presented in the figures). Approximate calculations from the current data support the view (Brasseur \& Wei [41], in non-rotating turbulence) that "distant" interactions with $s>10$ contribute relatively little to energy transfer. 


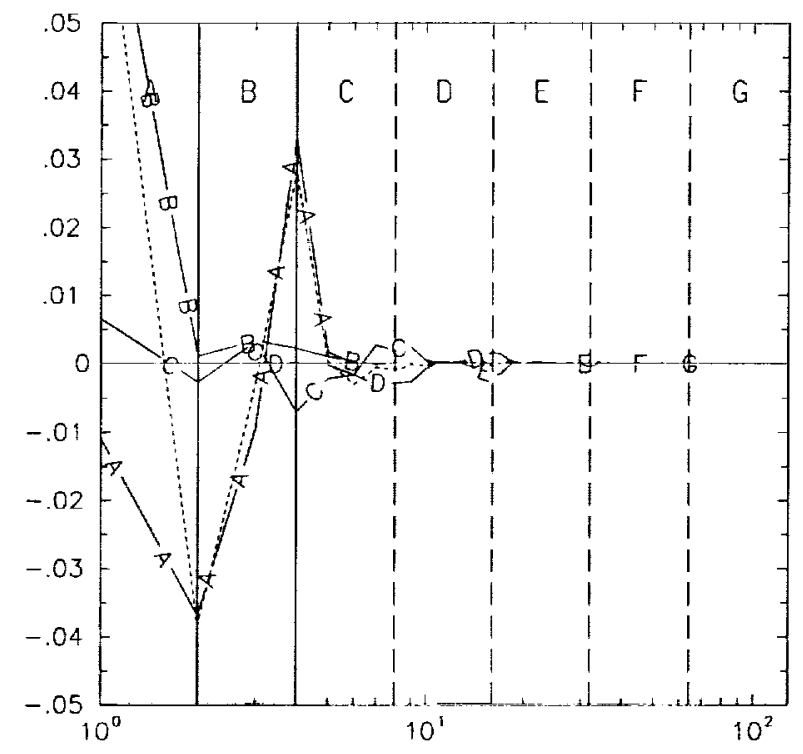

FIG. 19. Same as Fig.15, but for the perpendicular velocity components $T_{\alpha \alpha}(k \mid p)$. (Results are averaged over $\alpha=1$ and $\alpha=2$.)

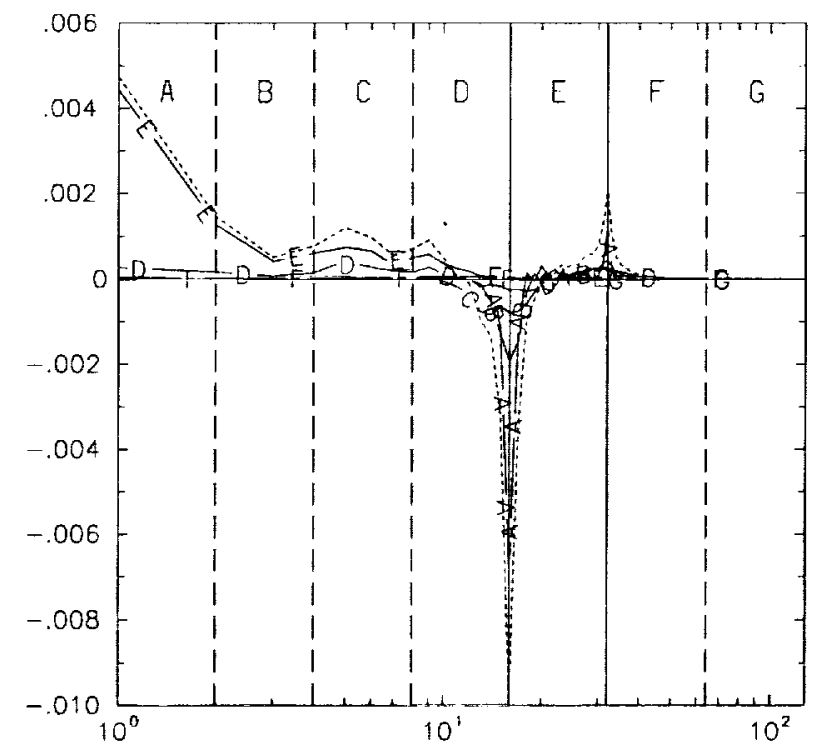

FIG. 20. Same as Fig.19, but for $16 \leq p<32$

Finally, it should be noted that although the anisotropizing tendency of spectral transfer is well established from Figs. 15 and 16, anisotropy characteristics in wavenumber space ultimately depend on a balance of competing terms (see Eq. 8). At high wavenumbers (where forcing is absent) we can write balance equations for the component spectra parallel and perpendicular to $\underline{\Omega}\left(E_{\| \mid}(k)\right)$ and $E_{\perp}(k)$ respectively) in the form

$$
\frac{d E_{\|}(k)}{d t}=T_{\|}(k)+A_{\|}(k)-D_{\|}(k)
$$

(with a similar equation for $d E_{\perp}(k) / d t$ ), where the symbols $T, A$ and $D$ represent transfer, rotation (Eq. 9) and dissipation contributions respectively. Numerical values recorded at several typical high wavenumber shells are listed in Table III. It can be seen that transfer and dissipation are dominant terms (of opposing 
signs), whereas the rotation term is almost negligible (although nonzero) by comparison. Whereas transfer and dissipation for the perpendicular component cancel each other almost completely, it is worth noting that in the parallel component transfer is consistently greater than dissipation. Furthermore, the overall rate of change $d E_{\|}(k) / d t$ is much greater than $d E_{\perp}(k) / d t$. Consequently, we may conclude that at high wavenumber anisotropic transfer is indeed stronger than the isotropizing effects of dissipation, with the net result being a departure from local isotropy under rotation.

4. Conclusions and discussion. In this paper we have used direct numerical simulations to study the response of initially isotropic turbulence subjected to uniform solid-body rotation in the presence of external energy input by numerical forcing. The grid resolution is $256^{3}$, initial Taylor scale Keynolds number is 140 (sufficient for a limited $k^{-5 / 3}$ inertial range in the energy spectrum), and rotation rates correspond to initial turbulent Rossby numbers of 0.0195 and 0.0039 . A major emphasis is to examine the detailed structure and anisotropy characteristics of spectral transfer, as well as the possibility of deviations from local isotropy at the small scales.

As expected, energy transfer and hence dissipation are greatly reduced by rotation. After a transient period, the kinetic encrgy increases approximately linearly with time as forcing energy input grows to exceed the loss by dissipation. Consistent with a prediction by Zhou [25], The energy spectrum attains a characteristic $k^{-2}$ scaling range at intermediate wavenumbers, although the Reynolds numbers are presumably not high enough for an accurate determination of parameters charactering a rotation-modified identical range.

Several measures of anisotropy are examined. Analysis of component budgets show that pressure-strain correlations and temporal oscillations due to rotation prevent the Reynolds stress tensor (which is a onepoint characteristic) from developing significant anisotropy over time. However, strong anisotropy is evident in (i) increased integral length scales along the axis of rotation, (ii) the high wavenumber part of the radial energy spectrum, and (iii) deviations of one-dimensional longitudinal and transverse spectra from isotropy relations at high wavenumbers. In addition, vorticity statistics exhibit anisotropic properties suggestive of the formation of slender vortical structures aligned with the axis of rotation.

Spectral transfer properties are studied in some detail, mainly in terms of triadic interactions in wavenumber space. As expected, rotation greatly weakens the energy cascade at the small scales. Considerable differences are found between the transfer characteristics of the velocity components parallel and perpendicular to the axis of rotation. The former has similarities with results in non-rotating turbulence and contributes dominantly to the reduced energy cascade. By contrast, the latter shows indications of reverse energy transfer from the intermediate scales towards the lowest wavenumbers in the spectrum, primarily as a result of triadic interactions of a local character.

Anisotropy of the energy spectrum at high wavenumbers is seen to be mainly a result of anisotropic transfer, although this is opposed by the isotropizing effects of the viscous dissipation. Essentially, whereas the large scales receive energy from forcing in all coordinate components, this energy is effectively removed to the small scales only in the component parallel to the axis of rotation.

In summary, we may conclude that the simulation results reported herein represent a situation in which rotation leads to the departure of the small scales from classical concepts of local isotropy. This is perhaps 
not a total surprise, since Kolmogorov's hypotheses, like most turbulence models have traditionally been formulated in non-inertial frames of reference without considering the effects of Coriolis forces. Nevertheless, these findings imply that subgrid-scale modeling procedures for rotating flows must take this anisotropy into account. For instance, it may be appropriate to use anisotropic filters to define the cutoff between resolved and subgrid motions, and to use general tensor definitions in subgrid eddy viscosity formulations.

Finally, we note that a similar suppression of spectral transfer for passive scalar fluctuations will most likely lead to reduced efficiency in turbulent mixing.

Acknowledgments. The computations were performed using IBM SP2 parallel supercomputers at NASA Ames and Langley Research Centers through the HPCCP/CAS Program. We also thank Professor Charles Speziale and Dr. Jackson Herring for their encouragement, and Dr. Jeffrey Chasnov for a valuable suggestion concerning the numerical methods.

Appendix. Analysis of early-time transient behavior. As noted in Sec. III.A (sec Fig. 1), the onset of rotation causes a marked decrease in both the kinetic energy and (especially) the disspation rate at early times. Here we address the physical and numerical causes for this transient behavior. Comparisons with decaying turbulence without forcing but subjected to rotation are also included.

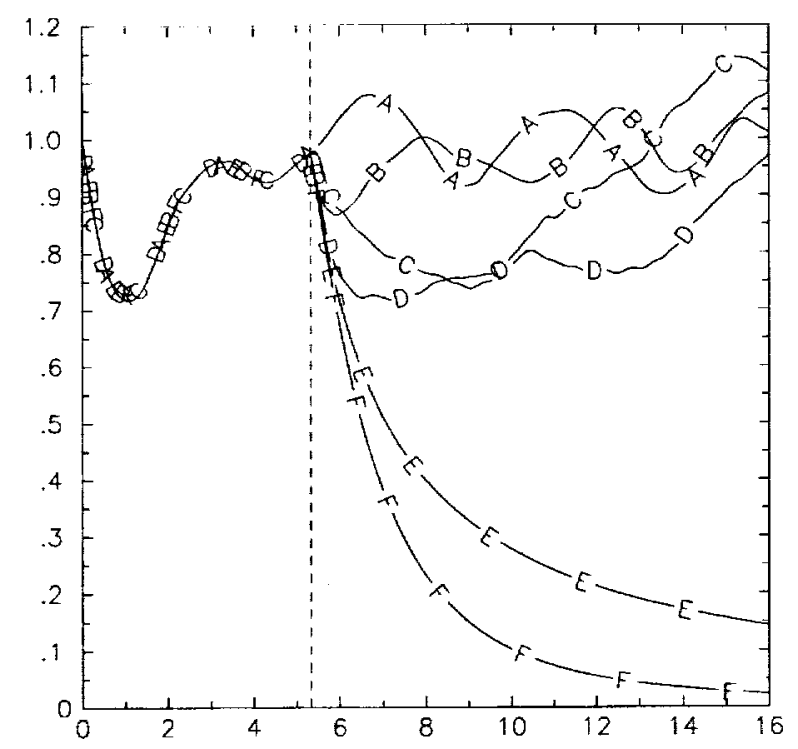

FIG. 21. Evolution of turbulence kinetic energy $K(t) / K(0)$ (normalized by initial values) verse $t / T_{E, 0}$ in the six sets of $64^{3}$ simulations discussed in Appendix. Lines $A$ to $F$ are for Cases $I$ to VI respectively, with simulation conditions as listed in Table II.

From a physical perspective, it is clear that both forcing and rotation have strong effects on the flow. It should also be noted that at any one time the effects of forcing on the kinetic energy are determined by the statistical correlation (or phase coupling in wavenumber space) between the instantaneous velocity field $\left(u_{i}\right)$ and the forcing acceleration $\left(f_{i}\right)$. In general, this correlation may be instantaneously positive, zero, or even negative. In this work $f_{i}$ is implemented as a stochastic process (Eswaran \& Pope [28]) via a computergenerated random number sequence. Consequently if a simulation is re-started afresh with a new "seed" 


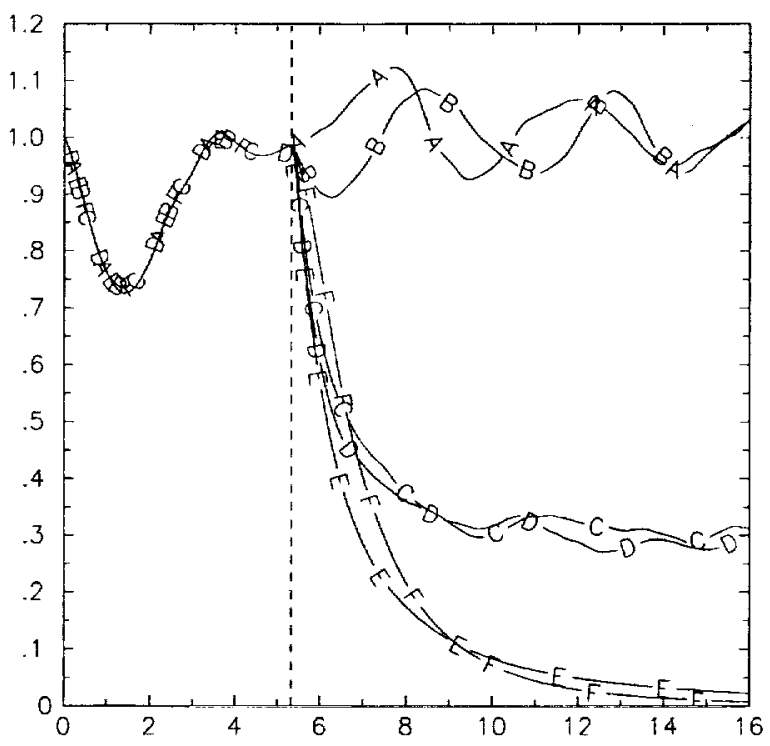

FIG. 22. Same as Fig.21, but for the dissipation rate $\epsilon(t) / \epsilon_{0}$

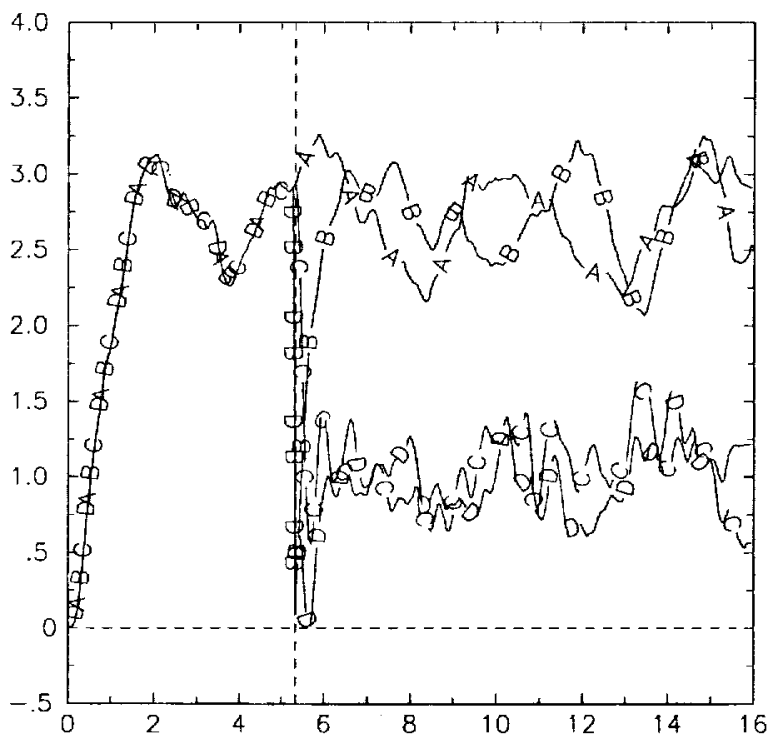

FIG. 23. Forcing energy input rate $\left\langle u_{i} f_{i}\right\rangle$ verse $t / T_{E, 0}$ for the first four cases (I to $I V$, as lines $A$ to $D$ ) in $T a b l e I I$.

for the random number sequence then $u_{i}$ and $f_{i}$ are expected to become (instantaneously) independent and hence the energy input rate would be effectively zero. Over time, a statistical coupling does develop between the velocity and the forcing, resulting ultimately in a positive energy input rate. Even then, because the forcing is applied only to the largest scales (of which there are only a limited number of samples in the solution domain), this energy input is subject to considerable statistical variability in time.

To illustrate these arguments it suffices to consider a set of simulations at lower grid-resolution, cach starting from the same initial conditions but performed with different parameters. Conditions for such a series of $64^{3}$ simulations in six different modes are listed in Table II. 
In all cases the initial conditions were taken from a previous simulation of forced stationary turbulence without rotation at Taylor-scale Reynolds number 38; and in the rotating cases the initial turbulent Rossby number is 0.069 . Furthermore in each case four independent realizations were carried out and the results averaged accordingly. Figures 21-23 show, respectively, the evolution of kinetic energy, dissipation rate, and forcing energy input (the latter excluding the unforced cases V and VI).

Cases I and II represent simulations of forced turbulence without rotation. In Case I the original random number sequence prior to the simulation restart $\left(t / T_{E, 0}=5.3\right.$, marked by vertical dashed line $)$ is continued, so that quantities vary smoothly with time without abrupt changes. Case II shows the effect of using a new random number sequence at re-start: the energy input rate drops to zero (as discussed above), causing a sudden drop of energy and dissipation. However, the effect of this numerical artifact is relatively short-lived, and within about one eddy-turnover time the energy input catches up to its former levels as a positive coupling is re-established between the velocity field and the forcing term. A similar effect of the random number sequences can be identified in the rotating simulations by comparing Cases III and IV.

Comparisons between cases I and III, and between II and IV, illustrate the effects of rotation alone. It may be seen that rotation causes a much stronger initial decay in energy and dissipation than that due to new random number sequences in the forcing scheme. At later times both the forcing energy input and dissipation rate maintain quasi-steady levels which are however much lower than those observed in the non-rotating cases.

In Cases V and VI the turbulence undergoes viscous decay as the forcing is removed. It is significant, however, that rotation causes a lesser reduction in kinetic encrgy overall and a sharper drop in dissipation rate in the earlier stages. This observation is consistent with the results of Speziale et al. [11] who showed that rotation reduces the decay rate of (unforced) isotropic turbulence - cssentially by shutting off the energy cascade to the smaller scales.

\section{REFERENCES}

[1] J. BARdina, J.H. FERziger, AND R.S. Rogallo, Effect of rotation on isotropic turbulence: Computation and modeling, J. Fluid Mech. 154, 1985, p. 321.

[2] N.N. MANSoUR, C. CAMBON, AND C.G. SPEzIALE, Theoretical and computational study of rotating isotropic turbulence, in Studies in Turbulence, eds. T.B. Gatski, S. Sarkar \& C.G. Speziale, SpringerVerlag, New York, 1992.

[3] C.G. SPEZIALE, Turbulence modeling in non-inertial frames of reference, Theor. Comput. Fluid Dyn. 1, 1989, p. 3.

[4] N.N. Mansour, C. Cambon, and C.G. Speziale, Single point modeling of initially isotropic turbulence under uniform rotation, in Annual Research Briefs, Center for Turbulence Research, Stanford University and NASA Ames Research Center, 1991.

[5] A.H. Hadid, N.N. MANsour, ANd O. Zeman, Single point modeling of rotating turbulent flows, Proceedings of the 1994 Summer Program, Center for Turbulence Research, Stanford University and NASA Ames Research Center, 1994.

[6] A. Mahalov AND Y. Zhou, Analytical and phenomenological studies of rotating turbulence, Phys. 
Fluids 8, 1996, p. 2138.

[7] R. Rubinstein, AND Y. Zhou, Analytical theory of the destruction terms in dissipation rate transport equations, Phys. Fluids 8, 1996, p. 3172.

[8] L. Jacquin, O. Leuchter, C. Cambon, ANd J. Mathieu, Homogeneous turbulence in the presence of rotation, J. Fluid Mech. 220, 1990, p. 1.

[9] R.A. WIGELAND, AND H.M. NAGIB, Grid-generated turbulence with and without rotation about the streamwise direction, IIT Fluids and Heat Transfer Rep. R. 78-1, Illinois Institute of Technology, 1978.

[10] C.G. Speziale AND R.M.C. So, Turbulence modeling and simulation. Technical Report No. AM-96015, Department of Aerospace and Mechanical Engineering, Boston University, 1996.

[11] C.G. Speziale, N.N. Mansour, AND R.S. Rogallo, The decay of isotropic turbulence in a rapidly rotating frame, Proc. of the 1987 Summer Program, Center for Turbulence Rescarch, Stanford University and NASA Ames Research Center, 1987.

[12] C. Cambon, N.N. Mansour, And K.D. Squires, Anisotropic structure of homogeneous turbulence subjected to uniform rotation, Proc. of the 1994 Summer Program, Center for Turbulence Research, Stanford University and NASA Ames Research Center, 1994.

[13] H.P. Greenspan, The Theory of Rotating Fluids, Cambridge University Press, 1968.

[14] M. Hossain, Reduction in the dimensionality of turbulence due to a strong rotation, Phys. Fluids 6 , 1994, p. 1077.

[15] J.G. BRASSEUR AND S. CoRrSin, Spectral evolution of the Navier-Stokes equations for low order couplings of Fourier modes, in Advances in Turbulence, eds. G. Comte-Bellot and J. Mathieu, Springer-Verlag, Berlin, 1987, p. 152.

[16] J.A. Domaradzki ANd R.S. Rogallo, Local energy transfer and non-local interactions in homogeneous, isotropic turbulence, Phys. Fluids A 2, 1990, p. 413. .

[17] Y. ZHOU, Interacting scales and energy transfer in isotropic turbulence, Phys. Fluids A 5, 1993, p. 2511.

[18] J.A. Domaradzki and W. LiU, Approximation of subgrid-scale energy transfer based on the dynamics of the resolved scales of turbulence, Phys. Fluids 7, 1995, p. 2025.

[19] Y. Zhou AND G. VAhala, Reformulation of recursive renormalization group based subgrid modeling of turbulence, Phys. Rev. E, 47, 1993, p. 2503.

[20] J.A. DomaradzKI AND E.M. SAIKI, A subgrid-scale model based on the estimation of unresolved scales of turbulence, Phys. Fluids 9, 1997, p. 2148.

[21] J.A. Domaradzki, P.K. Yeung, ANd Y. Zhou, A velocity estimation model for large eddy simulations, Proc. of the First AFOSR International Conf. on DNS/LES, Louisiana Tech University, Ruston, LA, 1997.

[22] C. CAMBon AND L. JACQUin, Spectral approach to non-isotropic turbulence subjected to rotation, J. Fluid Mech. 202, 1989, p. 295.

[23] C. CAmbon, N.N. Mansour, ANd F.S. GodefERd, Energy transfer in rotating turbulence, J. Fluid Mech. 337, 1997, p. 303.

[24] P.K. YEUnG AND Y. ZHOU, On the universality of the Kolmogorov constant in numerical simulations of turbulence, Phys. Rev. E 56, 1997, p. 1746.

[25] Y. ZHOU, A phenomenological treatment of rotating turbulence, Phys. Fluids 7, 1995, p. 2092.

[26] P.K. Yeung AND C.A. Moseley, A message-passing, distributed memory parallel algorithm for direct numerical simulation of turbulence with particle tracking, in Parallel Computational Fluid Dynamics, 
eds. A. Ecer et al. , Elsevier, Amsterdam, 1995, p. 473.

[27] R.S. Rogallo, Numerical experiments in homogeneous turbulence, NASA Tech. Memo. 81315, 1981.

[28] V. Eswaran AND S.B. Pope, An examination of forcing in direct numerical simulations of turbulence, Comput. \& Fluids 16, 1988, p. 257.

[29] L.M. SMith, J.R. Chasnov, and F. WALEFFe, The crossover from two- to three-dimensional turbulence, Phys. Rev. Lett. 77, 1996, p. 2467.

[30] O. ZEMAN, A note on the spectra and decay of rotating homogeneous turbulence, Phys. Fluids 6, 1994, p. 3221.

[31] G.K. BAtchelor, The Theory of Homogeneous Turbulence. Cambridge University Press, U.K., 1953.

[32] V.M. Canuto and M.S. Dubovikov, A dynamical model for turbulence. V. The effect of rotation, Phys. Fluids 9, 1997, p. 2132.

[33] J.L. Lumley AND G.R. Newman, The return to isotropy of homogeneous turbulence, J. Fluid Mech. 82, 1977, p. 161.

[34] N.N. Mansour, T.-H. ShiH, W.C. AND Reynolds, The effects of rotation on initially anisotropic homogeneous flows, Phys. Fluids A 3, 1991, p. 2421.

[35] W.C. REYNoldS AND S.C. KASSINOS, One-point modeling of rapidly deformed homogeneous turbulence, Proc. Roy. Soc. Lond. A 451, 1995, p. 87.

[36] K.D. Squires, J.R. Chasnov, N.N. Mansour, and C. Cambon, On the asymptotic similarity of rotating homogeneous turbulence, Proceedings of the 1994 Summer Program, Center for Turbulence Research, Stanford University and NASA Ames Research Center, 1994.

[37] Y. KimURA AND J.R. HerRING, Vortex formation in rotating stably stratified turbulence, Bull. Am. Phys. Soc. 41, No. 9, 1996, p. 1820.

[38] P.K. YEUnG AND J.G. BRAsSEUR, The response of isotropic turbulence to isotropic and anisotropic forcing at the large scales, Phys. Fluids A 3, 1991, p. 884.

[39] Y. Zhou, P.K. Yeung, and J.G. Brasseur, Scale disparity and spectral transfer in anisotropic numerical turbulence, Phys. Rev. E 53, 1996, p. 1261.

[40] P.K. YEUng, Multi-scalar spectral transfer in differential diffusion with and without mean scalar gradients, J. Fluid Mech. 321, 1996, p. 235.

[41] J.G. BRASSEUR AND C.-H. WEI, Interscale dynamics and local isotropy in high Reynolds number turbulence within triadic interactions, Phys. Fluids 6, 1994, p. 842. 
TABLE II

Terms (all in $10^{-5}$ ) in the spectral balance (Eq. 19) for velocity components parallel and perpendicular to the axis of ratation.

\begin{tabular}{lrrrr|rrrr}
$k$ & $T_{\|}(k)$ & $A_{\|}(k)$ & $-D_{\|}(k)$ & $d E_{\|}(k) / d t$ & $T_{\perp}(k)$ & $A_{\perp}(k)$ & $-D_{\perp}(k)$ & $d E_{\perp}(k) / \mathrm{dt}$ \\
60 & 34.10 & -1.123 & -29.72 & 3.257 & 6.532 & 0.561 & -6.946 & 0.147 \\
70 & 13.76 & -0.375 & -12.92 & 0.465 & 2.924 & 0.187 & -3.082 & -0.129 \\
80 & 6.376 & -0.131 & -5.691 & 0.554 & 1.381 & 0.065 & -1.411 & 0.035 \\
90 & 2.933 & -0.047 & -2.693 & 0.247 & 0.685 & 0.023 & -0.068 & 0.029
\end{tabular}

TABLE III

Forcing and ratation options for six sets of $64^{3}$ simulations discussed in the Appendix.

Use and mode of forcing Use of rotation

Case I With continuing random number sequence No rotation

Case II With new random number sequence No rotation

Case III With continuing random number sequence With rotation

Case IV With new random number sequence With rotation

Case V Forcing discontinued With rotation

$\begin{array}{lll}\text { Case VI } & \text { Forcing discontinued } & \text { No rotation }\end{array}$ 


\begin{tabular}{|c|c|c|c|}
\hline \multicolumn{3}{|c|}{ REPORT DOCUMENTATION PAGE } & $\begin{array}{l}\text { Form Approved } \\
\text { OMB No. 0704-0188 }\end{array}$ \\
\hline \multicolumn{4}{|c|}{ 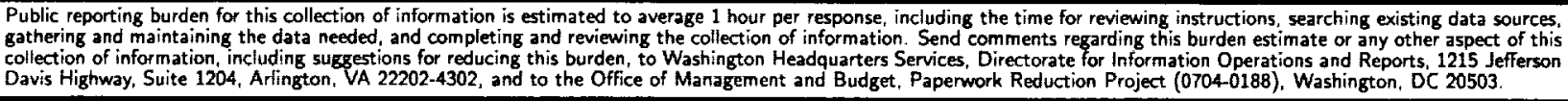 } \\
\hline 1. AGENCY USE ONLY(Leave blank) & $\begin{array}{l}\text { 2. REPORT DATE } \\
\text { August } 1998\end{array}$ & \multicolumn{2}{|c|}{$\begin{array}{l}\text { 3. REPORT TYPE AND DATES COVERED } \\
\text { Contractor Report }\end{array}$} \\
\hline \multicolumn{3}{|c|}{$\begin{array}{l}\text { 4. TITLE AND SUBTITLE } \\
\text { Numerical study of rotating turbulence with external forcing }\end{array}$} & \multirow[t]{2}{*}{$\begin{array}{l}\text { 5. FUNDING NUMBERS } \\
\text { C NAS1-19480 } \\
\text { C NAS1-97046 } \\
\text { WU } 505-90-52-01\end{array}$} \\
\hline \multicolumn{3}{|l|}{$\begin{array}{l}\text { 6. AUTHOR(S) } \\
\text { P.K. Yeung } \\
\text { Ye Zhou }\end{array}$} & \\
\hline \multicolumn{3}{|c|}{$\begin{array}{l}\text { 7. PERFORMING ORGANIZATION NAME(S) AND ADDRESS(ES) } \\
\text { Institute for Computer Applications in Science and Engineering } \\
\text { Mail Stop 403, NASA Langley Research Center } \\
\text { Hampton, VA } 23681-2199\end{array}$} & $\begin{array}{l}\text { 8. PERFORMING ORGANIZATION } \\
\text { REPORT NUMBER } \\
\text { ICASE Report No. } 98-39\end{array}$ \\
\hline \multicolumn{3}{|c|}{$\begin{array}{l}\text { 9. SPONSORING/MONITORING AGENCY NAME(S) AND ADDRESS(ES) } \\
\text { National Aeronautics and Space Administration } \\
\text { Langley Research Center } \\
\text { Hampton, VA } 23681-2199\end{array}$} & $\begin{array}{l}\text { 10. SPONSORING/MONITORING } \\
\text { AGENCY REPORT NUMBER } \\
\text { NASA/CR-1998-208702 } \\
\text { ICASE Report No. } 98-39\end{array}$ \\
\hline \multicolumn{4}{|c|}{$\begin{array}{l}\text { 11. SUPPLEMENTARY NOTES } \\
\text { Langley Technical Monitor: Dennis M. Bushnell } \\
\text { Final Report } \\
\text { Submitted to Physics of Fluids }\end{array}$} \\
\hline \multicolumn{2}{|c|}{$\begin{array}{l}\text { 12a. DISTRIBUTION/AVAILABILITY STATEMENT } \\
\text { Unclassified-Unlimited } \\
\text { Subject Category } 34 \\
\text { Distribution: Nonstandard } \\
\text { Availability: NASA-CASI (301)621-0390 }\end{array}$} & & 12b. DISTRIBUTION CODE \\
\hline \multicolumn{4}{|c|}{$\begin{array}{l}\text { 13. ABSTRACT (Maximum } 200 \text { words) } \\
\text { Direct numerical simulations at } 256^{3} \text { resolution have been carried out to study the response of isotropic turbulence } \\
\text { to the concurrent effects of solid-body rotation and numerical forcing at the large scales. Because encrgy transfer to } \\
\text { the smaller scales is weakened by rotation, energy input from forcing gradually builds up at the large scales, causing } \\
\text { the overall kinetic energy to increase. At intermediate wavenumbers the energy spectrum undergoes a transition } \\
\text { from a limited } k^{-5 / 3} \text { inertial range to } k^{-2} \text { scaling recently predicted in the literature. Although the Reynolds stress } \\
\text { tensor remains approximately isotropic and three-component, evidence for anisotropy and quasi- two-dimensionality } \\
\text { in length scales and spectra in different velocity components and directions is strong. The small scales are found to } \\
\text { deviate from local isotropy, primarily as a result of anisotropic transfer to the high wavenumbers. To understand } \\
\text { the spectral dynamics of this flow we study the detailed behavior of nonlinear triadic interactions in wavenumber } \\
\text { space. Spectral transfer in the velocity component parallel to the axis of rotation is qualitatively similar to that } \\
\text { in non-rotating turbulence; however the perpendicular component is characterized by a greatly suppressed energy } \\
\text { cascade at high wavenumbers and a local reverse transfer at the largest scales. The broader implications of this work } \\
\text { are briefy addressed. }\end{array}$} \\
\hline \multirow{2}{*}{\multicolumn{2}{|c|}{$\begin{array}{l}\text { 14. SUBJECT TERMS } \\
\text { direct numerical simulations; rotating turbulence; } \\
\text { anisotropy and quasi- two-dimensionality }\end{array}$}} & & $\begin{array}{c}\text { 15. NUMBER OF PAGES } \\
32\end{array}$ \\
\hline & & & $\begin{array}{c}\text { 16. PRICE CODE } \\
\mathrm{A} 03 \\
\end{array}$ \\
\hline $\begin{array}{l}\text { 17. SECURITY CLASSIFICATION } \\
\text { OF REPORT } \\
\text { Unclassificd }\end{array}$ & $\begin{array}{l}\text { 18. SECURITY CLASSIFICATION } \\
\text { OF THIS PAGE } \\
\text { Unclassified }\end{array}$ & $\begin{array}{l}\text { 19. SECURITY CLASSIFICATION } \\
\text { OF ABSTRACT }\end{array}$ & $\begin{array}{l}\text { 20. LIMITATION } \\
\text { OF ABSTRACT }\end{array}$ \\
\hline NSN 7540-01-280-5500 & & & $\begin{array}{l}\text { Standard Form 298(Rev. 2-89) } \\
\text { Prescribed by ANSI Std. Z39-18 } \\
\text { 298-102 }\end{array}$ \\
\hline
\end{tabular}

